\title{
Is dynamic contrast enhancement still necessary in multiparametric magnetic resonance for diagnosis of prostate cancer: a systematic review and meta-analysis
}

\author{
Zhen Liang ${ }^{1}$, Rui Hu${ }^{1}$, Yongjiao Yang ${ }^{2}$, Neng An ${ }^{2}$, Xiaoxin Duo ${ }^{3}$, Zheng Liu ${ }^{4}$, Shangheng Shi ${ }^{5}$ Xiaoqiang Liu ${ }^{1}$ \\ ${ }^{1}$ Department of Urology, Tianjin Medical University General Hospital, Tianjin 300000, China; ${ }^{2}$ Department of Urology, Tianjin Medical University \\ Second Hospital, Tianjin 300000, China; ${ }^{3}$ Department of Cardiology, The Second Hospital of Hebei Medical University, Shijiazhuang 050000, \\ China; ${ }^{4}$ Department of Rheumatology and Immunology, Tianjin Medical University General Hospital, Tianjin 300000, China; ${ }^{5}$ Department of \\ Transplantation, Affiliated Hospital of Medical College Qingdao University, Qingdao 266000, China \\ Contributions: (I) Conception and design: Z Liang, Y Yang, X Duo, Z Liu; (II) Administrative support: Z Liu; (III) Provision of study materials or \\ patients: R Hu, X Duo, X Liu; (IV) Collection and assembly of data: R Hu, Z Liu, Z Liang; (V) Data analysis and interpretation: R Hu, N An; (VI) \\ Manuscript writing: All authors; (VII) Final approval of manuscript: All authors. \\ Correspondence to: Prof. Xiaoqiang Liu, MD, PhD. Department of Urology, Tianjin Medical University General Hospital, Tianjin 300000 , China. \\ Email: 15233367861@163.com.
}

Background: The purpose of this study is to systematically review the literatures assessing the value of dynamic contrast enhancement (DCE) in the multiparametric magnetic resonance imaging (mpMRI) for the diagnosis of prostate cancer (PCa).

Methods: We searched Embase, PubMed and Web of science until January 2019 to extract articles exploring the possibilities whether the pre-biopsy biparametric magnetic resonance imaging (bpMRI) can replace the position of $\mathrm{mpMRI}$ in the diagnosis of $\mathrm{PCa}$. The sensitivity and specificity of bpMRI were all included. The study quality was assessed by QUADAS-2. Bivariate random effects meta-analyses and a hierarchical summary receiver operating characteristic plot were performed for further study through Revman 5 and Stata12.

Results: After searching, we acquired 752 articles among which 45 studies with 5,217 participants were eligible for inclusion. The positive likelihood ratio for the detection of PCa was 2.40 (95\% CI: 1.50-3.80) and the negative likelihood ratio was 0.31 (95\% CI: 0.18-0.53). The sensitivity and specificity were 0.77 (95\% CI: 0.73-0.81) and 0.81 (95\% CI: 0.76-0.85) respectively. Based on our result, pooled specificity demonstrated little difference between bpMRI and mpMRI [bpMRI, 0.81 (95\% CI, 0.76-0.85); mpMRI, 0.82 (95\% CI, 0.72-0.88); $\mathrm{P}=0.169]$. The sensitivity, however, indicated a significant difference between these two groups [bpMRI, 0.77 (95\% CI, 0.73-0.81); mpMRI, 0.84 (95\% CI, 0.78-0.89); P=0.001].

Conclusions: bpMRI with high b-value is a sensitive tool for diagnosing PCa. Consistent results were found in multiple subgroup analysis.

Keywords: Prostate cancer (PCa); biparametric; multiparametric; magnetic resonance imaging (MRI); contrast media; gadolinium; meta-analysis

Submitted Sep 28, 2019. Accepted for publication Jan 15, 2020.

doi: $10.21037 /$ tau.2020.02.03

View this article at: http://dx.doi.org/10.21037/tau.2020.02.03 


\section{Introduction}

Prostate cancer $(\mathrm{PCa})$ is the most commonly diagnosed disease in male around the world and its incidence and mortality have been increasing $(1,2)$. In last several years, multiparametric magnetic resonance imaging (mpMRI) has emerged as a valuable tool for several aspects of $\mathrm{PCa}$ management, including detection, staging, and treatment $(3,4)$. In order to standardize and diminish the variation in acquisition, interpretation, and reporting of prostate mpMRI, the European Society of Urogenital Radiology proposed the Prostate Imaging Reporting and Data System (PI-RADS) in 2012 (5). In December 2014, the updated and simplified PI-RADS version 2 (PI-RADSv2) was introduced to address the limitations and issues derived from the old version (3). It summarized the level of suspicion of PCa in a five-point scale based on mpMRI findings considering the combination of $\mathrm{T} 2$-weighted (T2W), diffusion-weighted imaging (DWI), and dynamic contrast-enhanced MRI [dynamic contrast enhancement (DCE)] (5). It is notable, however, in PI-RADSv2, DCEMRI is considered to play only a minor role in the detection of prostate tumors, and has a secondary role to T2W and DW MRI. Recent studies have demonstrated good accuracy of biparametric-MRI (bpMRI) - the combination of T2-weighted imaging and DWI, used for tumor detection when evaluated with PSA (6-8).

DCE-MRI serves to show the perfusion parameters of tissues. It gathers information about the vascularity of tissues by assessing the signal intensity of overtime after administration of gadolinium contrast material. Greer et al. (9) indicated that DCE-MRI added extra benefits to the application of PI-RADSv2 because abnormal DCEMRI findings increased the cancer detection rate in every PI-RADSv2 categories 2, 3, 4, and 5. Puech et al. (10) considered DCE as one of the cornerstones of mpMRI for its improvement in detection and evaluation of $\mathrm{PCa}$ aggressiveness. On the other hand, those who advocated the nonuse of DCE suggested that bpMRI has several advantages over mpMRI, such as shorter examination time, lower risk of allergy associated with gadoliniumbased contrast agents $(7,11)$. Aydin et al. (12) indicated both highly vascularized BPH nodules and prostatitis can lead to increased vessel enhancement, which may cause low specificity of mpMRI. Although the updated version of PI-RADS maps out guidelines of the interpretation of DCE-MRI and acquisition processing for imaging, Berman et al. (13) pointed out there were still sources of variability, such as the application of $3 \mathrm{~T}$ scanners thus it is difficult for DCE-MRI to reproduce results across centers. In our current study, based on quantitative data, a comparison has been drawn between bpMRI and mpMRI through systematic review and meta-analysis.

\section{Methods}

\section{Literature search}

The protocol for systematic review was written according to the Cochrane Handbook for Systematic Review of Interventions version 5.1.0 (14). We searched PubMed, Embase, Web of Science to make a head to head comparison between bpMRI and mpMRI in the diagnosis of PCa, and our search strategy was as follows: (prostate cancer OR prostatic cancer OR prostate neoplasm OR prostatic neoplasm OR prostate tumor OR prostatic tumor OR prostate carcinoma OR prostatic carcinoma OR PCa) AND (magnetic resonance imaging OR MRI OR MR) AND (biparametric OR bp OR T2-weighted image and DWI OR T2-weighted imaging and DWI) until January 2019. Handsearching of the reference lists of included studies was also performed to identify other relevant articles.

\section{Study selection}

The original studies can only be included in our network meta-analysis by meeting all the following requirements: (I) the study is published in English; (II) the available data is sufficient enough to calculate the diagnostic sensitivity and specificity of bpMRI; (III) the pathology results were provided by prostatectomy or prostate biopsy; (IV) the reported data is adequate for constructing $2 \times 2$ contingency tables with at least 10 patients. Narrative reviews, observational studies, editorials, letters comments, opinion pieces and methodological reports were all excluded. The relevant articles were selected by two researchers independently and disagreements were resolved by discussion.

Methodological quality of the included studies was evaluated by two authors independently using the same criteria as described in the Cochrane Manual for Systematic Intervention Reviews 5.2 to guarantee the quality of studies. Each item was scored as either low, high or unclear risk of bias. 


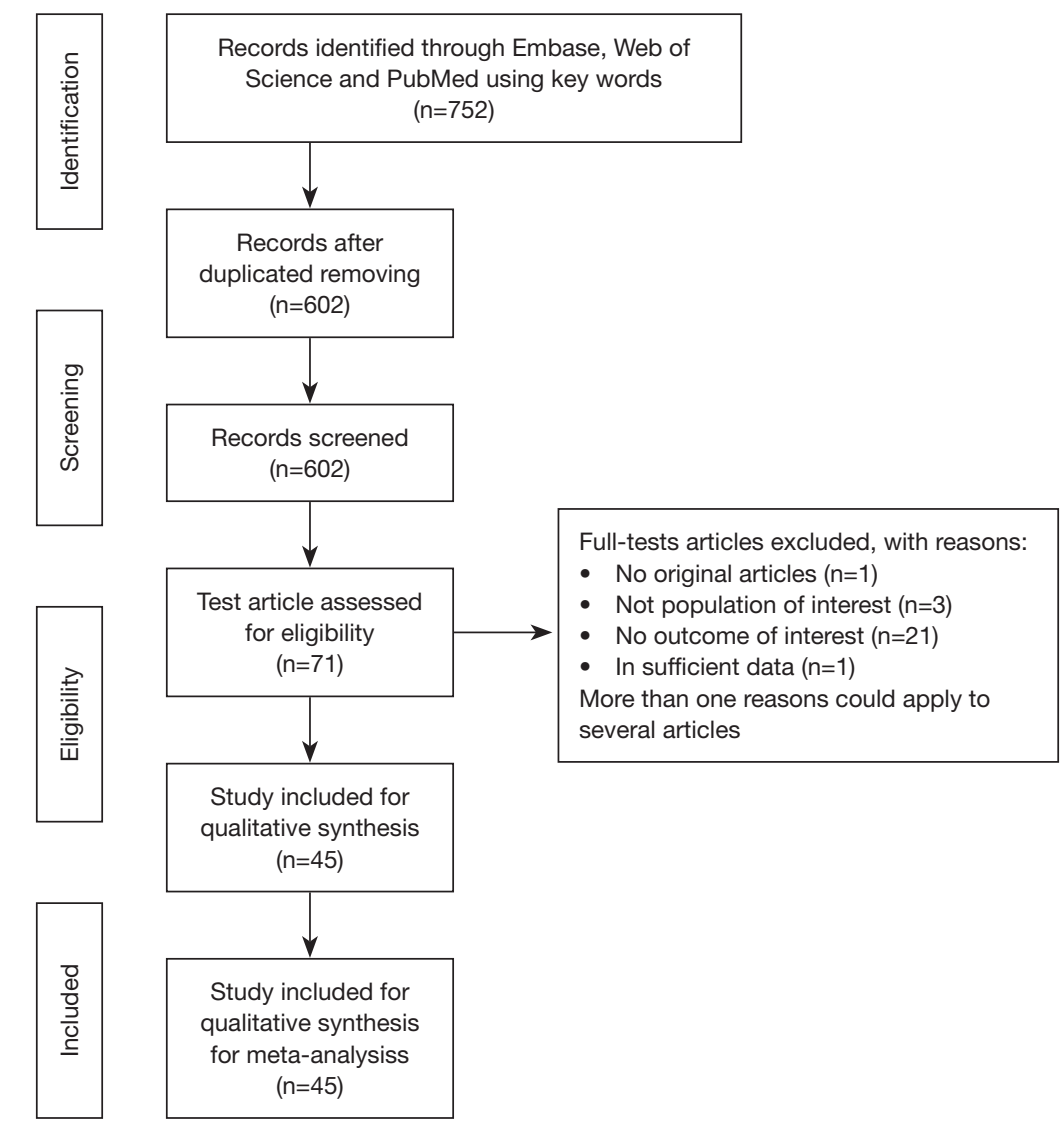

Figure 1 Flowchart summarizes selection process toward final group of studies analyzed.

\section{Statistical analysis}

Collection of results data for the quantitative synthesis was processed through Open Meta-analyst (15). All statistical analyses were conducted with the Midas module in Stata 13.1 (Stata Corporation, College Station, TX, USA). The sensitivity rate $\mathrm{TP} /(\mathrm{TP}+\mathrm{FN}) \times 100 \%$ and specificity rate $\mathrm{TN} /(\mathrm{TN}+\mathrm{FP}) \times 100 \%$ were calculated and two forest plots were generated side by side: one for specificity and the other for sensitivity. A bivariate random effects regression was performed to calculate several primary outcomes, including diagnostic likelihood ratio positive (DLR+), diagnostic likelihood ratio negative (DLR-), and diagnostic OR (DOR) pooled sensitivity, specificity, with corresponding $95 \%$ CIs (16). The summary receiver operating characteristic curve (SROC) was used to evaluate the predictive value of each scoring system. Deek's funnel plot was conducted to detect publication bias, with $P<0.05$ suggesting publication bias. Heterogeneity was valued with the Higgins-Thompson $I^{2}$ method and the Chi-square. The significant heterogeneity was indicated by $\mathrm{P}$ value $<0.05$ and $\mathrm{I}^{2}>50 \%$ (17). Subgroup analysis was accomplished if there was significant heterogeneity.

\section{Results}

\section{Study selection}

The electronic databases search yielded 752 titles and abstracts, among which 602 studies were selected to be fully reviewed; after excluding 362 duplicates and 240 conference abstracts, reviews, case reports and letters to journal editors, 71 studies were assessed for eligibility. The details of study selection are demonstrated in Figure 1. A total of 45 studies were included in the final analysis.

The sample size ranged from 20 to 1,063 , with a total of 5,217 patients included in our study. The involved 45 cohorts were carried out in the United States, Egypt, Switzerland, Germany, Denmark, France, Korea, Canada. Belgium, Japan, Finland, Austria, United States, Brazil, 
Italy, Spain and Turkey respectively. Among them 15 were $(8,18-31)$ prospective studies and 30 were retrospective studies. The publication period of these studies was from 2005 to 2018. The characteristics of included studies are presented in Tables 1,2. The age range of men was from 41 to 87 years (average 65.8). Across all studies, the PSA value ranged from $0.1-935.5 \mathrm{ng} / \mathrm{mL}$. The definition of clinically significant prostate cancer (csPCa) is also varied considerably.

A total of $22(8,18-20,27,29,31,32,35,37-39,42-44,48-50$, $55-58)$ studies were performed on biopsy-naive patients, and $4(7,24,32,48)$ studies reported on a mixed cohort (patients with previous prostate biopsy or no biopsy experience). The reference standard was based on radical prostatectomy in 23 $(11,18,21-23,25,26,28,34,37-39,42,44-46,48,51,53-56,59)$ studies, transperineal template saturation biopsy in 3 $(7,20,38)$ studies, targeted in-bore MRI-guided biopsy in $2(7,33)$ studies, MRI-ultrasound fusion guided biopsy in $5(32,35,41,43,47)$ studies. Patients of $24(18,21$ $26,28,30,31,33,36,37,39,41,42,45,48,50,51,55,57-59)$ included studies underwent MRI with a $1.5 \mathrm{~T}$ scanner, and $19(7,8,11,19,20,27,29,34,38,40,41,43,46,47,49,52-54,56)$ studies applied 3.0T scanner. Twenty-three $(8,11,19-25,27-$ $29,31,33,36-38,41,42,44,47,50,57)$ studies used endorectal coil. High $b$ values $\left(\geq 1,400 \mathrm{~s} / \mathrm{mm}^{2}\right)$ were applied in 11 $(7,8,30,38,41,43,46,49,52-54)$ studies and low b values $\left(<1,400 \mathrm{~s} / \mathrm{mm}^{2}\right)$ in 34 studies. Per-patient analysis was performed in $12(7,8,32,35,37,38,48-52,56)$ studies, and perlesion analysis in 33 studies.

\section{Assessment of study quality and publication bias}

The Quality Assessment of Diagnostic Accuracy Studies tool (QUADAS) was conducted to evaluate the quality of the study. The risk of bias for, index test, patient selection, flow and timing, reference standard, as well as the concerns for applicability were displayed in Figure 2. As for patient selection, $14(8,25,26,31,32,34,42-44,46,49,50,52,55)$ studies had high risk of bias as consecutive enrollment was not applied or mentioned in their articles. Regarding the index test domain, $7(18,21-23,42,49,55)$ studies had high risk of bias because instead of prespecifying the cutoff value for diagnosing the presence of $\mathrm{PCa}$, they established the values based on ROC curve analysis. Thirteen $(18,19,24$, $27,29,31,32,35,38,47,51,56,59)$ studies did not provide enough proof that whether the MRI screening results were interpreted by assessors blinded to the biopsy results. In case of reference standard, radical prostatectomy or MRI-
TRUS fusion-guided targeted biopsy were considered as the low risk reference standard. Other methods such as TRUSguided biopsy or transperineal biopsy were considered to be of high risk. Therefore, the risk of bias in the reference standard was high in $12(8,19,20,29-31,36,40,49,50,57,58)$ studies. About flow and timing, 8 (7,18,26,28,38,46,55,56) studies had high risk of bias because all included patients did not undergo the same reference standard, some underwent radical prostatectomy while others underwent TRUS- or MRI-guided biopsy. Twelve $(8,19,20,29-31$, $36,40,49,50,57,58)$ studies had unclear bias for the interval between the reference standard and MRI was not provided. For applicability, $4(18,33,36,50)$ studies have high risk of bias since T2W or DWI sequence was used solely instead of combining them together.

Little publication bias was detected by Begg rank correlation (with continuity correction) and Egger's linear regression test of funnel plot asymmetry in this metaanalysis with a $\mathrm{p}$ value of 0.55 for the slope coefficient (Figure 3).

\section{Overall diagnostic accuracy}

The result of the including researches was listed in Figure 4. The sensitivity of bpMRI for distinguishing cancerous and noncancerous specimen ranged from $45 \%$ to $99 \%$, and the specificity ranged from $37 \%$ to $100 \%$. The pooled sensitivity was 0.77 (95\% CI: $0.73-0.81$ ) with heterogeneity $\left(\mathrm{I}^{2}=93.55, \mathrm{P}=0.00\right)$ and a pooled specificity of $0.81(95 \%$ CI: $0.76-0.85)$ with heterogeneity $\left(\mathrm{I}^{2}=95.73, \mathrm{P}=0.00\right)$. On the other hand, the sensitivity of bpMRI for distinguishing csPCa and insignificant $\mathrm{PCa}$ (insPCa) specimen ranged from $49 \%$ to $96 \%$, and its specificity was ranged from $34 \%$ to $88 \%$. The pooled sensitivity was 0.78 (95\% CI: $0.66-$ $0.87)$ with heterogeneity $\left(\mathrm{I}^{2}=96.14, \mathrm{P}=0.00\right)$ and a pooled specificity of 0.77 (95\% CI: 0.66-0.85) with heterogeneity $\mathrm{I}^{2}=98.00, \mathrm{P}=0.00$ ) (Figure 5). The performance of bpMRI for carcinoma in different locations was also evaluated in our present study. Concerning the peripheral zone the sensitivity of bpMRI was $75 \%$ (95\% CI: $0.67-0.82$ ) ranging from $32-91 \%$ with heterogeneity $\left(\mathrm{I}^{2}=88.64, \mathrm{P}=0.00\right)$, and the specificity was $81 \%$ (95\% CI: $0.73-0.87)$ ranging from $45-98 \%$ with heterogeneity $\left(\mathrm{I}^{2}=92.76, \mathrm{P}=0.00\right)$ (Figure 6). The sensitivity of bpMRI for transition zone was $80 \%(95 \%$ CI: $0.73-0.85)$ ranging from $72-100 \%$ with heterogeneity $\left(\mathrm{I}^{2}=70.13, \mathrm{P}=0.00\right)$, the specificity was $80 \%$ (95\% CI: $0.70-0.87)$ ranging from $50-91 \%$ with heterogeneity $\left(\mathrm{I}^{2}=92.95, \mathrm{P}=0.00\right)$ (Figure 7). The summary AUC was 0.86 


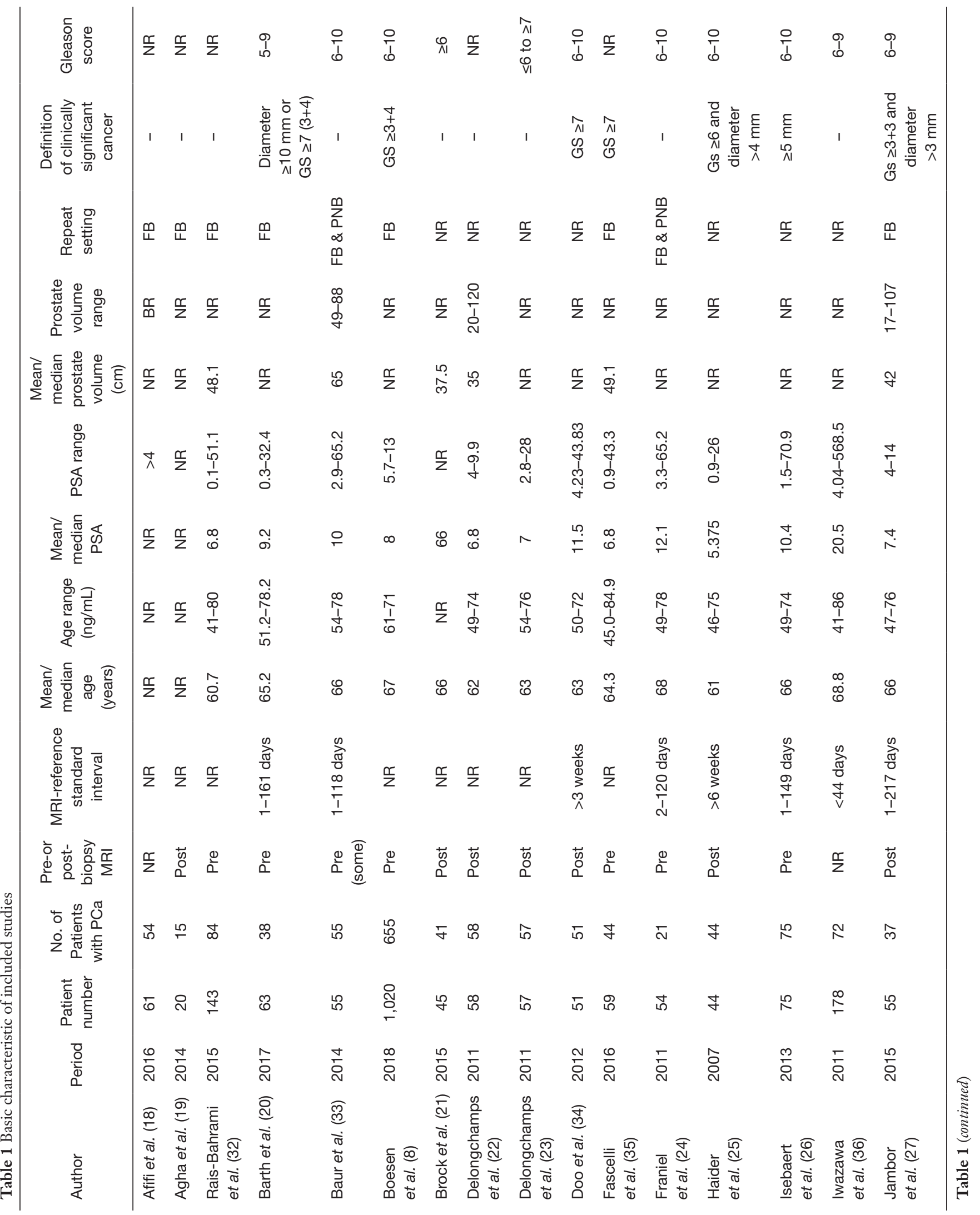




\begin{tabular}{|c|c|c|c|c|c|c|c|c|c|c|c|c|c|c|}
\hline 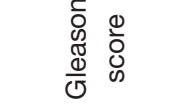 & $\stackrel{\varphi}{\Lambda}$ & $\hat{0}$ & $\frac{0}{7}$ & $\frac{\mathfrak{r}}{z}$ & $\stackrel{N}{N}_{1}$ & ํㅜㅁ & $\begin{array}{l}0 \\
1\end{array}$ & $\frac{0}{1}$ & $\begin{array}{l}9 \\
1\end{array}$ & $\begin{array}{l}0 \\
1\end{array}$ & $\mathscr{N}_{1}$ & $\frac{0}{1}$ & $\frac{\mathfrak{r}}{z}$ & $\begin{array}{l}\text { p } \\
0\end{array}$ \\
\hline 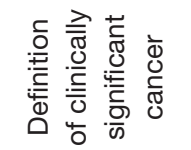 & 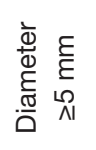 & I & 1 & 1 & 1 & 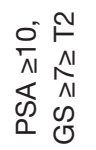 & 1 & 1 & 1 & 1 & $\frac{\mathfrak{r}}{z}$ & 1 & 1 & 1 \\
\hline 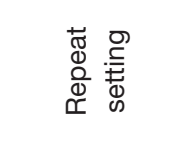 & $\underset{4}{\mathscr{L}}$ & $\mathscr{\mathscr { P }}$ & 田 & $\frac{\mathfrak{r}}{z}$ & 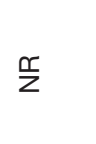 & $\begin{array}{l}m \\
\sum z \\
0 \\
\infty \\
p \\
u\end{array}$ & $\sum_{a}^{m}$ & 呆 & 呆 & $\frac{\mathbb{r}}{z}$ & 呆 & 甼 & 呆 & 愛 \\
\hline 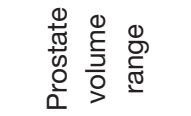 & $\frac{\mathfrak{r}}{z}$ & $\begin{array}{l}\text { 임 } \\
\frac{1}{1} \\
\frac{1}{2}\end{array}$ & $\frac{\mathfrak{q}}{z}$ & $\frac{\mathfrak{r}}{z}$ & $\stackrel{\frac{\Upsilon}{z}}{z}$ & $\begin{array}{l}\stackrel{\circ}{\circ} \\
\stackrel{1}{1} \\
\stackrel{0}{\leftarrow}\end{array}$ & $\frac{\mathfrak{q}}{z}$ & $\frac{\Upsilon}{z}$ & $\frac{\mathfrak{r}}{z}$ & $\frac{\mathfrak{r}}{z}$ & $\begin{array}{l}0 \\
\vdots \\
1 \\
\infty\end{array}$ & $\frac{\Upsilon}{z}$ & $\frac{\mathfrak{r}}{z}$ & 孚 \\
\hline 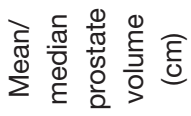 & $\frac{\mathfrak{q}}{z}$ & \& & 愛 & $\frac{\Upsilon}{z}$ & 愛 & กิ & $\frac{\Upsilon}{z}$ & 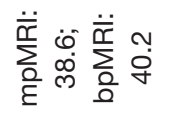 & $\frac{\mathfrak{r}}{z}$ & $\frac{\mathfrak{r}}{z}$ & \& & $\frac{\mathfrak{r}}{z}$ & 愛 & 愛 \\
\hline 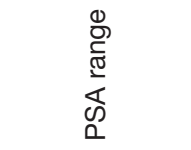 & 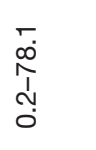 & 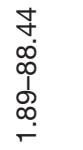 & $\underset{\stackrel{+}{T}}{\stackrel{+}{0}}$ & 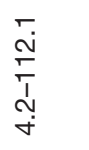 & $\begin{array}{l}\stackrel{0}{+} \\
\stackrel{1}{+} \\
\dot{+}\end{array}$ & 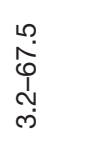 & $\begin{array}{l}\stackrel{0}{\infty} \\
\stackrel{1}{\sim} \\
\stackrel{\Gamma}{r}\end{array}$ & $\frac{\Upsilon}{z}$ & $\begin{array}{l}\stackrel{\circ}{0} \\
\stackrel{0}{1} \\
\stackrel{1}{\sim} \\
\stackrel{1}{\leftarrow}\end{array}$ & 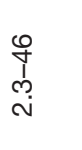 & $\begin{array}{l}0 \\
\stackrel{0}{1} \\
\infty \\
\infty \\
\infty\end{array}$ & $\begin{array}{l}m \\
\stackrel{0}{0} \\
1 \\
\infty \\
\stackrel{N}{N}\end{array}$ & $\begin{array}{l}\infty \\
\infty \\
1 \\
\infty \\
o \\
\infty \\
\infty\end{array}$ & $\begin{array}{l}\stackrel{\infty}{0} \\
\stackrel{m}{p} \\
\stackrel{m}{r}\end{array}$ \\
\hline 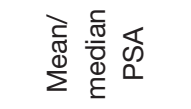 & ما & $\stackrel{+}{\oplus}$ & $\begin{array}{l}0 \\
\infty\end{array}$ & $\underset{\Gamma}{\Gamma}$ & $\hat{\omega}$ & $\wedge$ & 음 & 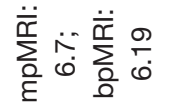 & $\begin{array}{l}\stackrel{0}{\circ} \\
\stackrel{0}{\circ}\end{array}$ & $\stackrel{\infty}{\infty}$ & $\stackrel{\mathscr{r}}{\dot{f}}$ & 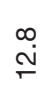 & $\begin{array}{l}\frac{10}{20} \\
\frac{1}{6}\end{array}$ & ָै \\
\hline 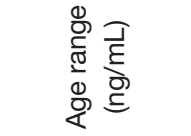 & $\begin{array}{l}\stackrel{g}{1} \\
\stackrel{\sim}{Y}\end{array}$ & $\frac{\mathfrak{r}}{z}$ & $\begin{array}{l}\infty \\
1 \\
⿱ 亠 乂 \\
\mathscr{y}\end{array}$ & \begin{tabular}{l}
\multirow{1}{0}{} \\
0 \\
0 \\
0
\end{tabular} & $\stackrel{\frac{\Upsilon}{z}}{2}$ & 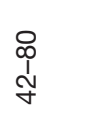 & $\begin{array}{l}\hat{1} \\
\hat{y}\end{array}$ & $\frac{\mathfrak{Y}}{z}$ & $\begin{array}{l}0 \\
\hat{1} \\
\stackrel{\infty}{q}\end{array}$ & 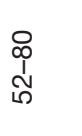 & $\frac{\mathfrak{n}}{z}$ & $\begin{array}{l}\stackrel{\infty}{\uparrow} \\
\stackrel{9}{\sigma}\end{array}$ & $\begin{array}{l}\infty \\
1 \\
1 \\
10\end{array}$ & \begin{tabular}{l}
0 \\
$\hat{1}$ \\
\multirow{f}{*}{}
\end{tabular} \\
\hline 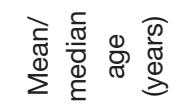 & ஸ̊ & 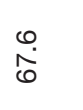 & R & 8 & $\widehat{\widehat{\theta}}$ & ஜ & $\Xi$ & 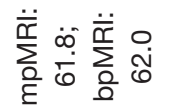 & ¿ٌ & $\stackrel{\infty}{0}$ & $\frac{\mathfrak{n}}{z}$ & กิ & 8 & $\widetilde{ఠ}$ \\
\hline 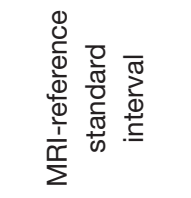 & $\begin{array}{l}\frac{\infty}{2} \\
\frac{\pi}{0} \\
\infty \\
\infty \\
\frac{1}{0}\end{array}$ & $\frac{\mathfrak{r}}{z}$ & 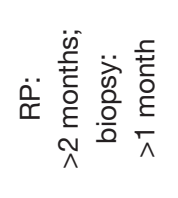 & 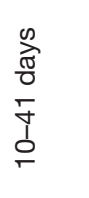 & 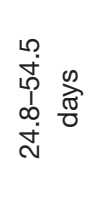 & $\begin{array}{l}\text { n } \\
\frac{0}{0} \\
8 \\
0 \\
\frac{0}{1} \\
\infty \\
\stackrel{N}{N}\end{array}$ & 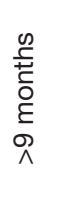 & 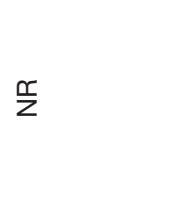 & 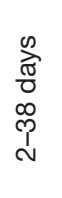 & 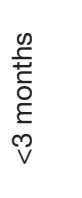 & 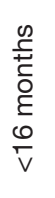 & $\frac{\mathfrak{r}}{z}$ & 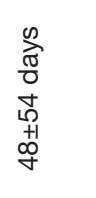 & $\frac{\mathfrak{r}}{z}$ \\
\hline 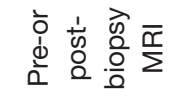 & $\stackrel{\Phi}{\underline{Q}}$ & $\stackrel{\varrho}{2}$ & $\begin{array}{l}\text { पू } \\
0\end{array}$ & $\stackrel{\varrho}{\underline{\Sigma}}$ & $\begin{array}{l}\text { पू } \\
0\end{array}$ & $\stackrel{\Xi}{0}$ & $\stackrel{\Phi}{\underline{\Sigma}}$ & $\stackrel{0}{0}$ & 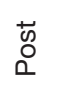 & $\begin{array}{l}\text { पू } \\
0 \\
0\end{array}$ & $\stackrel{\varrho}{0}$ & $\stackrel{\varrho}{\check{\Sigma}}$ & 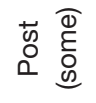 & पू \\
\hline 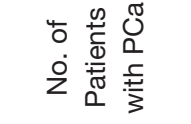 & $\stackrel{N}{N}$ & $\stackrel{\stackrel{\infty}{\oplus}}{\Gamma}$ & $\overline{\mathrm{N}}$ & ஜ्ల & 䓃 & 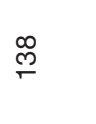 & $\stackrel{\varphi}{r}$ & $\stackrel{N}{N}$ & $\tilde{~}$ & t5 & $\stackrel{\infty}{0}$ & ొల & @) & $\stackrel{\Im}{\Im}$ \\
\hline 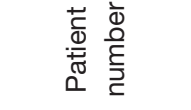 & $\begin{array}{l}\mathscr{L} \\
\stackrel{2}{2}\end{array}$ & $\stackrel{\mathscr{N}}{N}$ & $\bar{\sim}$ & నొ & 芯 & 疋 & ஜ్ల & م & $\tilde{\omega}$ & L5 & $\stackrel{\infty}{\leftarrow}$ & $\stackrel{m}{m}$ & ₹ & $\stackrel{\sim}{\Im}$ \\
\hline 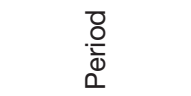 & $\stackrel{m}{i}$ & $\stackrel{\circ}{\stackrel{N}{N}}$ & $\bar{\Sigma}$ & 울 & $\stackrel{+}{\stackrel{N}{N}}$ & $\hat{i}$ & $\stackrel{+}{\stackrel{N}{N}}$ & $\hat{\grave{n}}$ & $\underset{\sim}{\stackrel{\sim}{O}}$ & 仓̊ & $\hat{\grave{N}}$ & $\stackrel{\Gamma}{\stackrel{N}{N}}$ & $\stackrel{\sim}{\stackrel{N}{*}}$ & $\bar{\Sigma}$ \\
\hline $\begin{array}{l}\frac{1}{2} \\
\frac{1}{5} \\
\frac{1}{4}\end{array}$ & 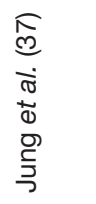 & 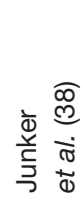 & 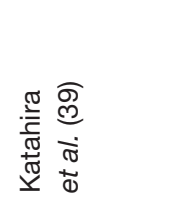 & 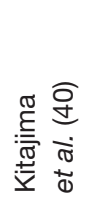 & 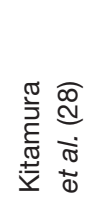 & 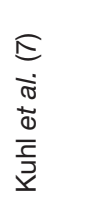 & 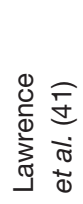 & 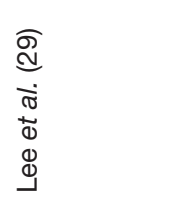 & 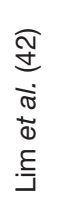 & 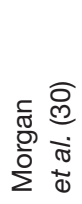 & 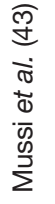 & 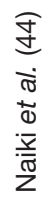 & 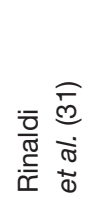 & 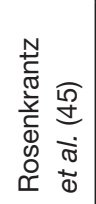 \\
\hline
\end{tabular}




\begin{tabular}{|c|c|c|c|c|c|c|c|c|c|c|c|c|c|c|c|}
\hline 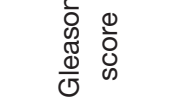 & $\stackrel{N}{N}_{1}$ & $\frac{\mathfrak{r}}{z}$ & $\frac{\Upsilon}{z}$ & $\begin{array}{l}p \\
1\end{array}$ & $\frac{0}{1}$ & $\frac{0}{1}$ & $\frac{\Upsilon}{z}$ & $\begin{array}{l}p \\
1 \\
0\end{array}$ & $\begin{array}{l}0 \\
1\end{array}$ & $\begin{array}{l}\infty \\
1 \\
0\end{array}$ & $\begin{array}{l}\infty \\
1 \\
0\end{array}$ & $\frac{\Upsilon}{z}$ & $\frac{0}{1}$ & 寽 & $\begin{array}{l}\text { p } \\
0\end{array}$ \\
\hline 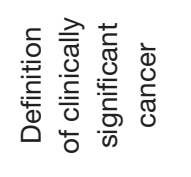 & $\begin{array}{l}\widehat{N} \\
\mathbb{N} \\
C\end{array}$ & I & 1 & 1 & 1 & 1 & $\begin{array}{l}\hat{\Lambda} \\
0 \\
0\end{array}$ & 1 & 1 & 1 & 1 & 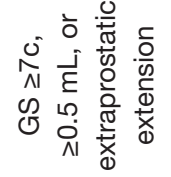 & 1 & 1 & 1 \\
\hline 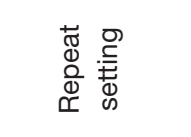 & $\frac{\mathfrak{r}}{z}$ & $\begin{array}{l}m \\
\sum \\
0 \\
\infty \\
\infty \\
w\end{array}$ & 呆 & ஜ & 艺 & $\frac{\mathfrak{r}}{z}$ & 愛 & 愛 & $\frac{\mathfrak{r}}{z}$ & $\frac{\mathfrak{r}}{z}$ & 呆 & 呆 & $\underset{\mathscr{L}}{\mathscr{P}}$ & 呆 & 愛 \\
\hline 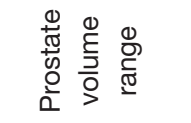 & $\frac{\mathfrak{r}}{z}$ & $\frac{\mathfrak{r}}{z}$ & $\frac{\mathfrak{r}}{z}$ & $\frac{\Upsilon}{z}$ & $\frac{\mathfrak{Y}}{z}$ & $\frac{\mathfrak{r}}{z}$ & $\begin{array}{l}\mathscr{N} \\
\stackrel{N}{W} \\
\text { N }\end{array}$ & $\frac{\tilde{c}}{z}$ & $\frac{\mathfrak{r}}{z}$ & $\frac{\mathfrak{r}}{z}$ & $\frac{\mathfrak{r}}{z}$ & $\frac{\Upsilon}{z}$ & $\frac{\Upsilon}{z}$ & $\begin{array}{l}\stackrel{1}{\infty} \overline{\grave{N}} \\
\stackrel{\sim}{\circ}\end{array}$ & 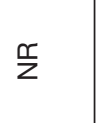 \\
\hline 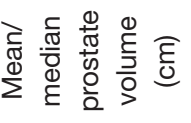 & $\frac{\mathfrak{c}}{z}$ & $\underset{\text { D̦ }}{\stackrel{0}{0}}$ & $\frac{\Re}{z}$ & $\begin{array}{l}\text { తి } \\
\text { ชิ }\end{array}$ & $\frac{\Upsilon}{z}$ & $\frac{\Upsilon}{z}$ & 8 & $\frac{\Upsilon}{z}$ & $\frac{\Upsilon}{z}$ & $\frac{\Upsilon}{z}$ & $\frac{\mathfrak{c}}{z}$ & $\frac{\Upsilon}{z}$ & $\frac{\Upsilon}{z}$ & $\begin{array}{c}\stackrel{\infty}{g} \\
\stackrel{8}{q}\end{array}$ & $\frac{\Upsilon}{z}$ \\
\hline 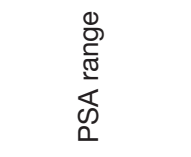 & 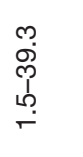 & $\frac{\Upsilon}{z}$ & 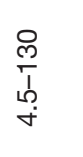 & $\frac{\mathfrak{r}}{z}$ & $\begin{array}{l}+ \\
o \\
0 \\
0 \\
0 \\
\dot{+}\end{array}$ & 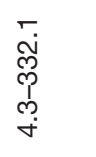 & 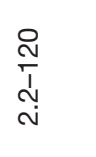 & $\begin{array}{l}\stackrel{g}{+} \\
\text { o } \\
\text { N }\end{array}$ & $\begin{array}{l}\stackrel{9}{+} \\
\stackrel{9}{i}\end{array}$ & 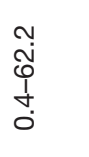 & 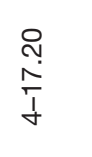 & 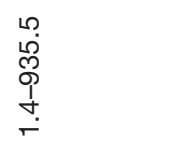 & 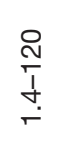 & \begin{tabular}{l}
$\infty$ \\
\multirow{1}{0}{} \\
$\stackrel{1}{1}$ \\
$\stackrel{0}{0}$
\end{tabular} & 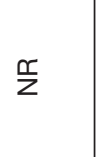 \\
\hline 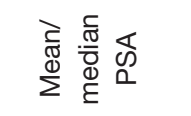 & $\stackrel{\infty}{\oplus}$ & $\stackrel{\circ}{\circ}$ & $\stackrel{\infty}{\grave{N}}$ & $\begin{array}{l}\infty \\
\infty \\
\infty\end{array}$ & $\begin{array}{l}\infty \\
@ \\
0\end{array}$ & $\stackrel{\nabla}{\stackrel{\sigma}{\sigma}}$ & $\stackrel{\nabla}{\ulcorner}$ & in & in & m & $\stackrel{ナ}{\stackrel{\leftrightarrow}{\wedge}}$ & $\sigma$ & $\stackrel{+}{\sigma}$ & $\stackrel{\leftrightarrow}{\leftarrow}$ & $\frac{\Upsilon}{z}$ \\
\hline 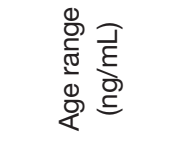 & $\begin{array}{l}\stackrel{\infty}{1} \\
\infty_{0}^{\infty}\end{array}$ & $\frac{\Upsilon}{z}$ & $\begin{array}{l}0 \\
0 \\
0 \\
0 \\
0\end{array}$ & 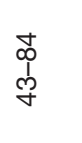 & $\begin{array}{l}\stackrel{2}{0} \\
b \\
b\end{array}$ & $\begin{array}{l}\widehat{\infty} \\
0 \\
\\
i n\end{array}$ & 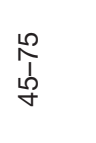 & $\begin{array}{l}\hat{A} \\
i \\
i\end{array}$ & $\begin{array}{l}\hat{1} \\
\text { ò }\end{array}$ & 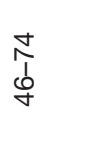 & 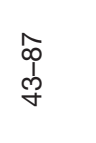 & 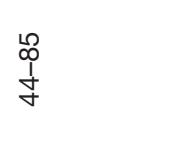 & 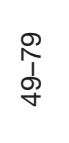 & $\begin{array}{l}\stackrel{2}{1} \\
1 \\
0 \\
\wp\end{array}$ & $\begin{array}{l}\bar{\infty} \\
1 \\
\sim \\
\end{array}$ \\
\hline 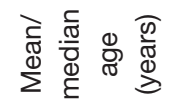 & $\begin{array}{l}\stackrel{1}{0} \\
\dot{0}\end{array}$ & 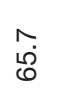 & $\bar{R}$ & 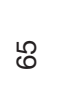 & $\mathscr{\wp}$ & 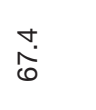 & $\mathscr{0}$ & $\begin{array}{l}n \\
0 \\
0\end{array}$ & 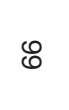 & $\stackrel{\infty}{\infty}$ & $\begin{array}{l}\stackrel{0}{0} \\
\widetilde{0}\end{array}$ & $\mathscr{8}$ & 8 & 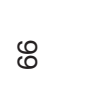 & $\stackrel{\infty}{\infty}$ \\
\hline 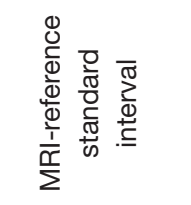 & 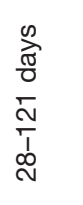 & 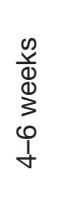 & 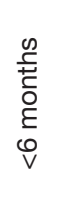 & 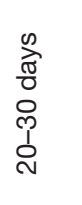 & 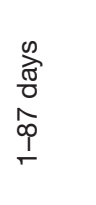 & 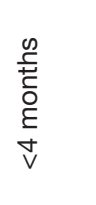 & 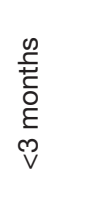 & 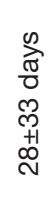 & $\frac{\mathscr{q}}{z}$ & 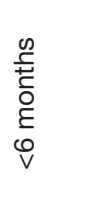 & 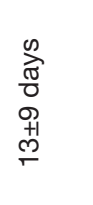 & 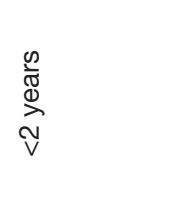 & $\frac{\Upsilon}{z}$ & $\begin{array}{l}0 \\
\Phi \\
\Phi \\
3 \\
0 \\
0 \\
0\end{array}$ & 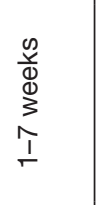 \\
\hline 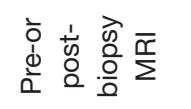 & $\begin{array}{l}\text { पे } \\
\text { o } \\
0\end{array}$ & $\begin{array}{l}\text { प्⿹ } \\
\text { O }\end{array}$ & $\begin{array}{l}\text { प्⿹ } \\
\text { O }\end{array}$ & $\stackrel{\varrho}{0}$ & $\stackrel{0}{2}$ & $\stackrel{0}{2}$ & 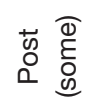 & $\begin{array}{l}\vec{m} \\
0 \\
0\end{array}$ & $\begin{array}{l}\text { 艹 } \\
0 \\
0\end{array}$ & $\begin{array}{l}\text { प्⿹ } \\
\text { O }\end{array}$ & $\stackrel{0}{0 .}$ & 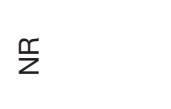 & $\stackrel{\Phi}{\Delta}$ & $\begin{array}{l}\text { "ू. } \\
\text { O }\end{array}$ & $\begin{array}{l}\text { "艹 } \\
\text { O }\end{array}$ \\
\hline 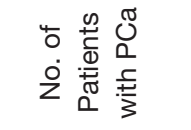 & 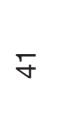 & $\stackrel{10}{\rightleftharpoons}$ & $\hat{m}$ & ले & $\stackrel{\infty}{m}$ & $\underset{\forall}{\forall}$ & $\stackrel{\infty}{0}$ & ○ & M & 5 & $\stackrel{\infty}{\infty}$ & $\stackrel{\infty}{\circ}$ & $\bar{N}$ & $\hat{m}$ & $\stackrel{\sim}{N}$ \\
\hline 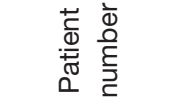 & テ & $\stackrel{\mathscr{N}}{N}$ & 8 & న & 요 & $\infty$ & 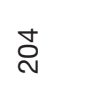 & ○ & $\stackrel{M}{N}$ & 5 & & $\stackrel{\mathscr{f}}{N}$ & ঙ & $\hat{m}$ & $\stackrel{\sim}{N}$ \\
\hline $\begin{array}{l}.0 \\
\frac{.0}{\overline{0}} \\
0 .\end{array}$ & స̃ & $\stackrel{+}{\circ}$ & ஜ̊ & $\stackrel{0}{\stackrel{N}{N}}$ & $\bar{i}$ & ڤ્̀ & $\stackrel{0}{\stackrel{N}{\alpha}}$ & 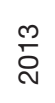 & 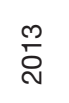 & స్⿱ & 울 & $\hat{\bar{N}}$ & $\bar{i}$ & $\stackrel{\infty}{\circ}$ & ஜे \\
\hline $\begin{array}{l}\frac{1}{0} \\
\frac{0}{5} \\
\frac{1}{2}\end{array}$ & 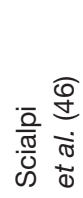 & 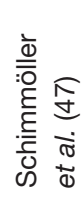 & 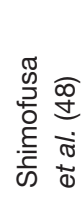 & 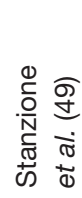 & 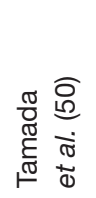 & 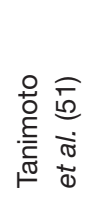 & 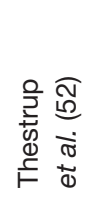 & 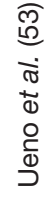 & 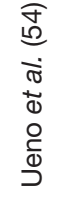 & 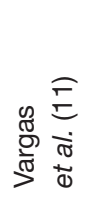 & 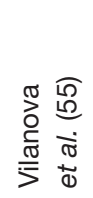 & 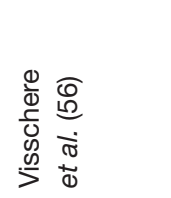 & 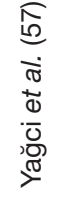 & 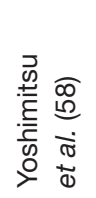 & 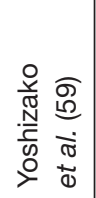 \\
\hline
\end{tabular}




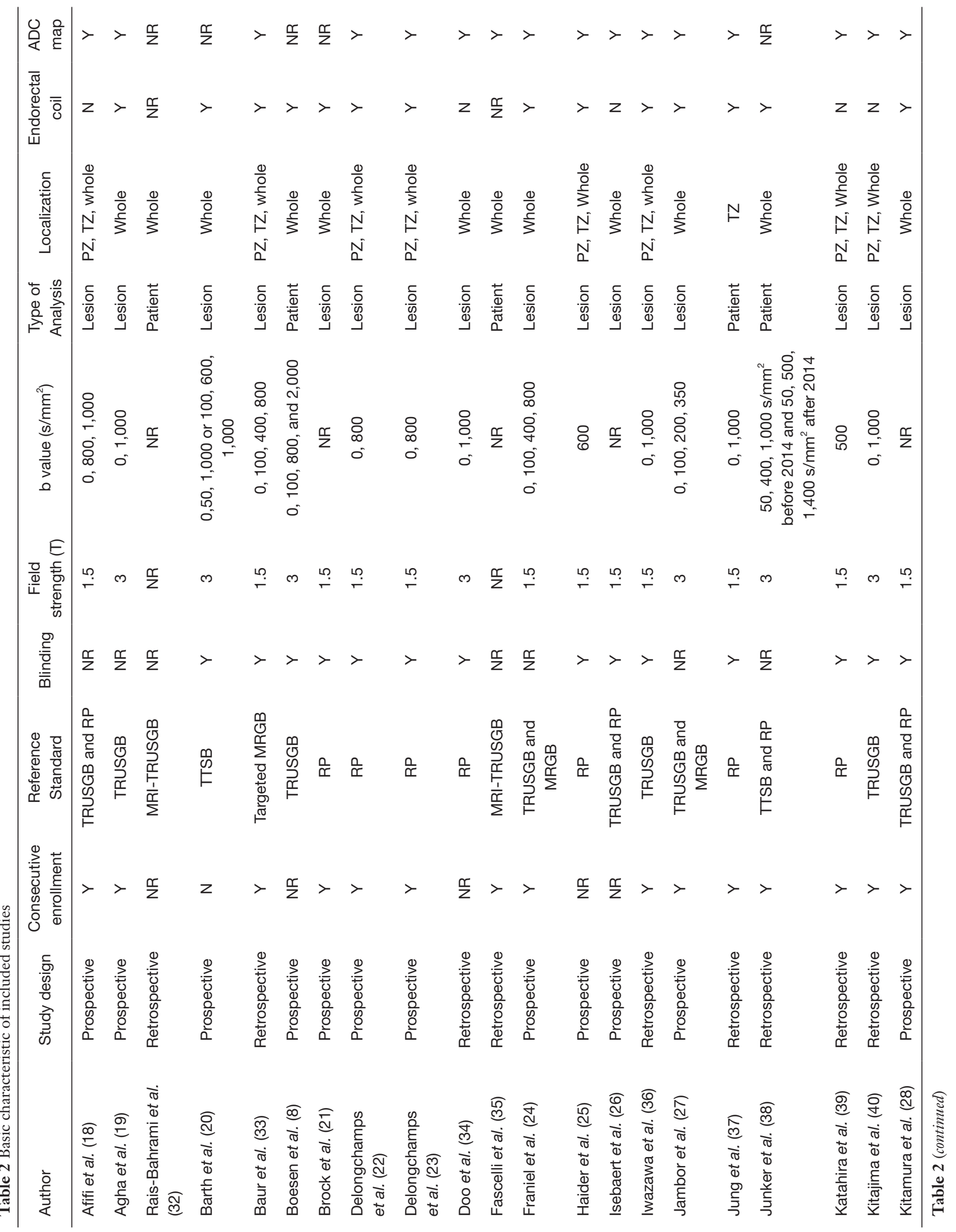




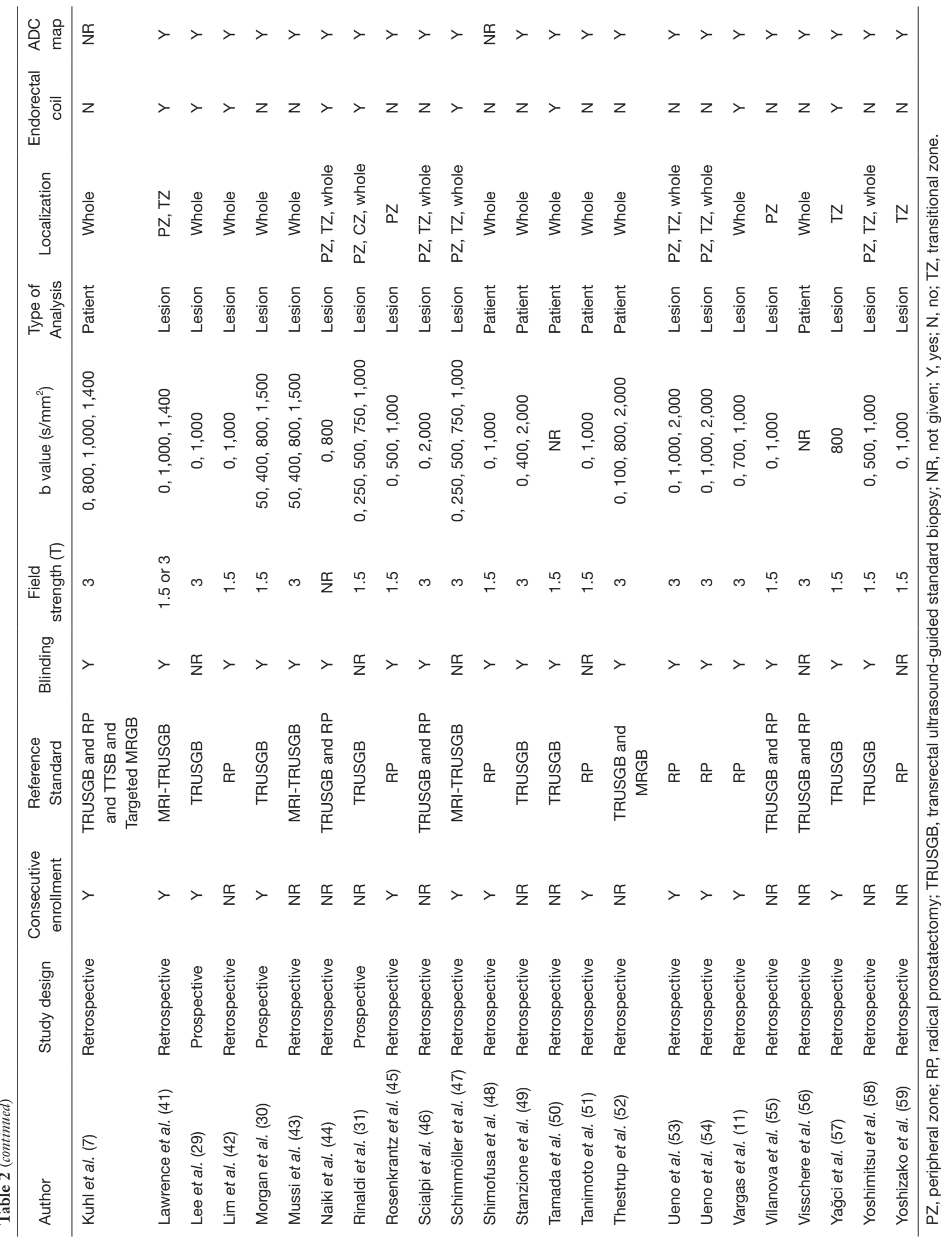




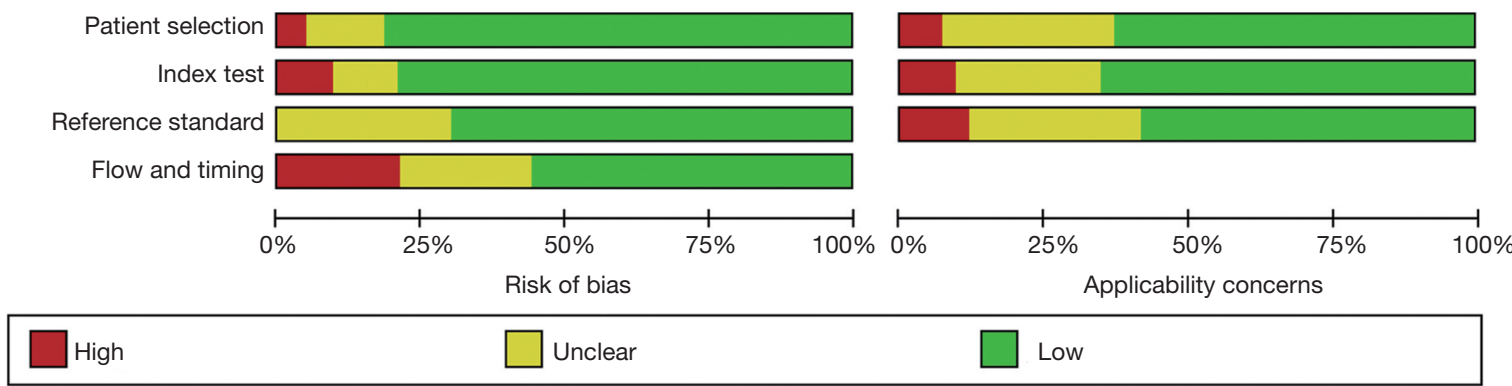

Figure 2 Chart shows summary of results of methodologic quality analysis of 45 studies in meta-analysis according to Quality Assessment of Diagnostic Accuracy Studies 2.

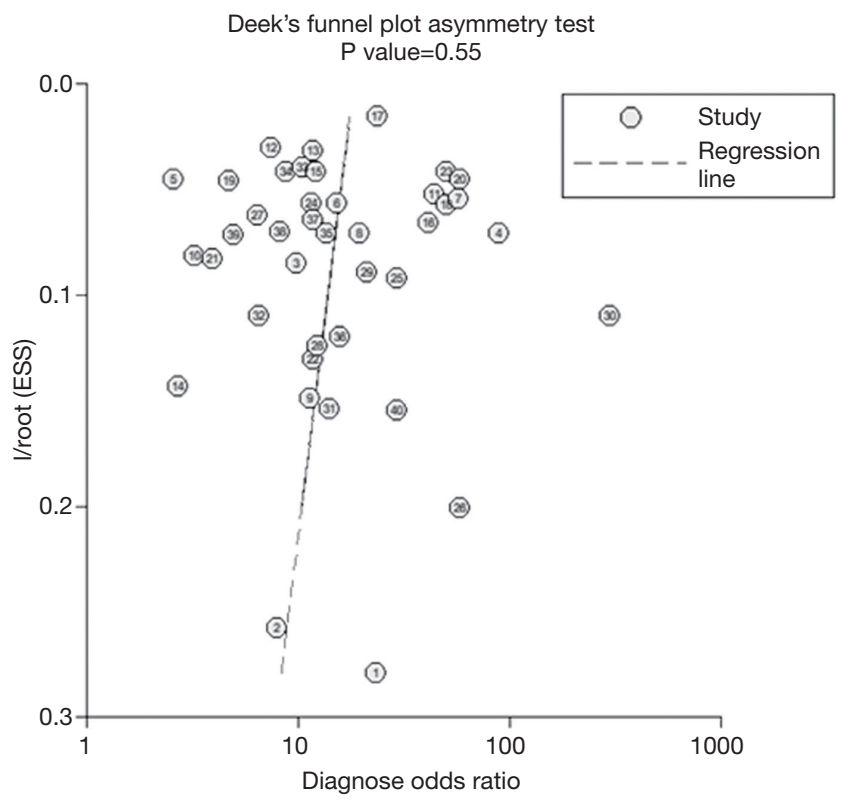

Figure 3 Plot results of Deeks funnel plot asymmetry test $(\mathrm{P}=0.55)$ show log odds ratios for visualization of publication bias

for overall cancer and 0.84 for csPCa which is similar to the performance of mpMRI $(0.90,0.83$ for overall $\mathrm{PCa}$ and csPCa respectively) (Figures 8,9). For the cancer located at the peripheral zone, the summary AUC of bpMRI was 0.85 (Figure 10A), while the AUC was 0.86 for transition zone cancer (Figure 10B). In addition, the overall positive LR and negative LR for the overall PCa 4.10 (95\% CI: 3.30-5.10) and 0.28 (95\% CI: 0.24-0.33), respectively. As for csPCa, the positive LR and negative LR were 3.40 (95\% CI: 2.44.9) and 0.29 (95\% CI: 0.18-0.45) respectively, and DOR, 15 (95\% CI, 11-20) for PCa, 12 (95\% CI, 6-22) for csPCa. The overall positive LR and negative LR for the peripheral zone cancer were 3.90 (95\% CI: 2.70-5.60) and 0.31 (95\%
CI: $0.23-0.40)$. For the transitional zone cancer, the overall positive LR and negative LR were 3.90 (95\% CI: 2.60-5.80) and 0.25 (95\% CI: 0.19-0.34) respectively. As for DOR, 13 (95\% CI, 8-21) for peripheral zone cancer, 15 (95\% CI, 9-27) for transitional zone cancer.

\section{Subgroup analyses and head-to-bead comparison}

Subgroup analysis was conducted based on study design, patient enrollment, localization the coil application, magnetic strength, b values, reference standard, blind method application and unit for analysis. Results of all subgroup analysis were summarized in Table 3. In accordance with the above results, the distinction among included studies could be explained as a source of the heterogeneity for the diagnosis of $\mathrm{PCa}$, and our result revealed that all the factors mentioned above accounted for the heterogeneity of sensitivity while none of them had an impact on specificity.

Our studies provided head-to-head comparison between bpMRI and mpMRI. As a result, the pooled specificity demonstrated little difference between bpMRI and mpMRI [bpMRI, 0.81 (95\% CI, 0.76-0.85); mpMRI, 0.82 (95\% CI, $0.72-0.88) ; \mathrm{P}=0.169$ ]. The sensitivity, however, indicated a significant difference between these two groups [bpMRI, 0.77 (95\% CI, 0.73-0.81); mpMRI, 0.84 (95\% CI, $0.78-$ 0.89); $\mathrm{P}=0.001]$ (Figures 4,11).

\section{Discussion}

Overall, we found very considerable diagnostic accuracy and precision for detection of PCa using bpMRI. Based on our assays, pooled sensitivity of bpMRI was $7 \%$ lower than that of mpMRI with statistical difference. Although the high sensitivity means higher confidence that a negative 

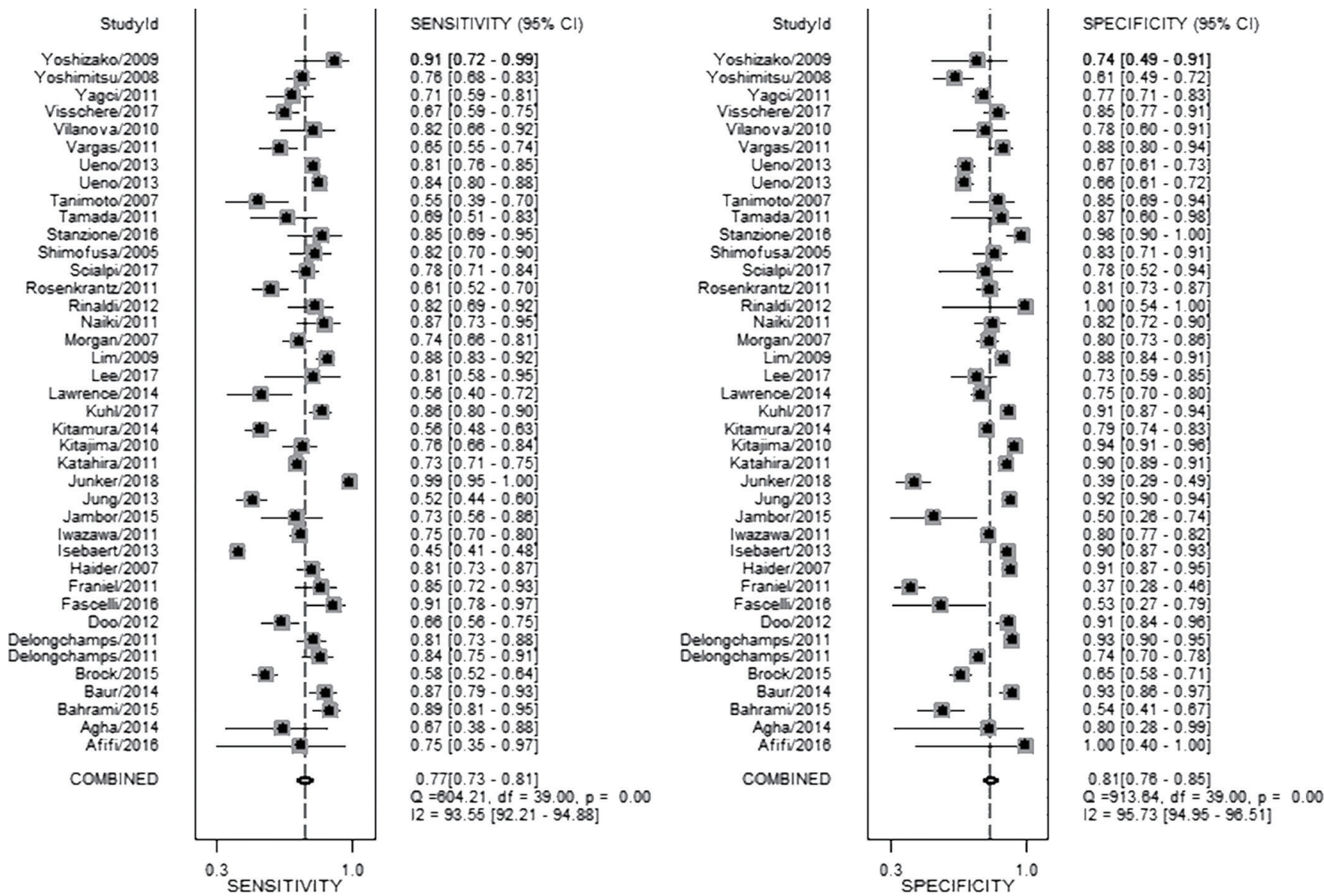

Figure 4 Coupled forest plots show pooled estimates of sensitivity and specificity of biparametric MRI for overall cancer.

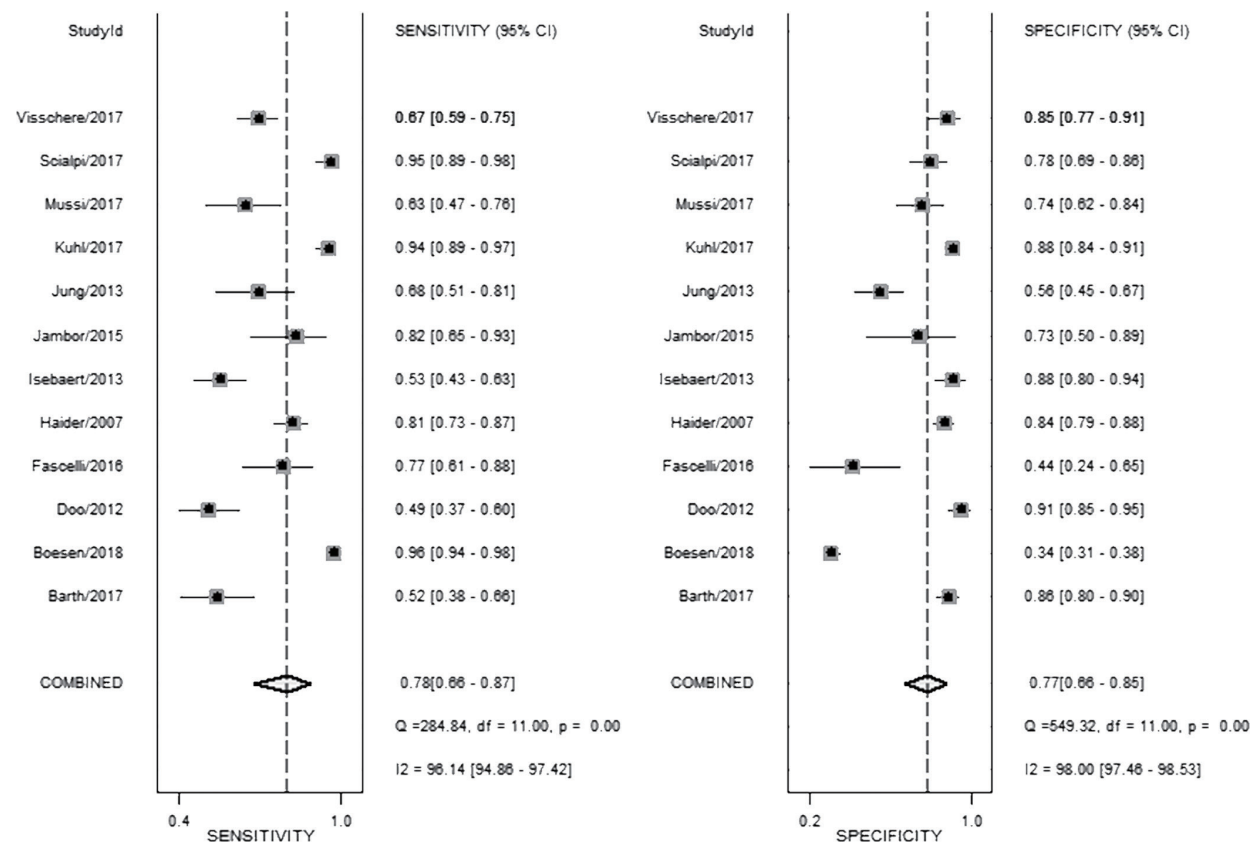

Figure 5 Coupled forest plots show pooled estimates of sensitivity and specificity of biparametric MRI for clinically significant cancer. 

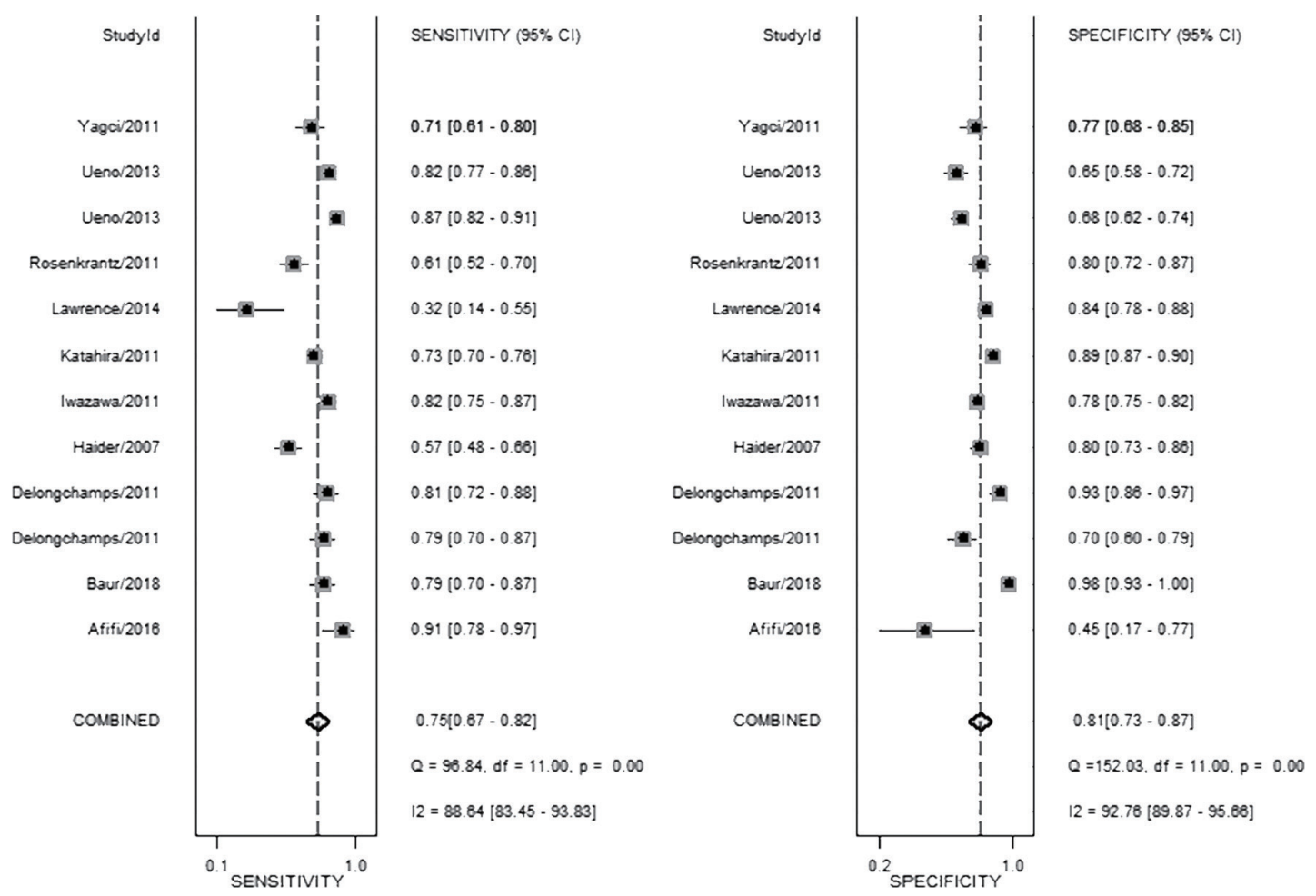

Figure 6 Coupled forest plots show pooled estimates of sensitivity and specificity of biparametric MRI for cancer located at peripheral zone.

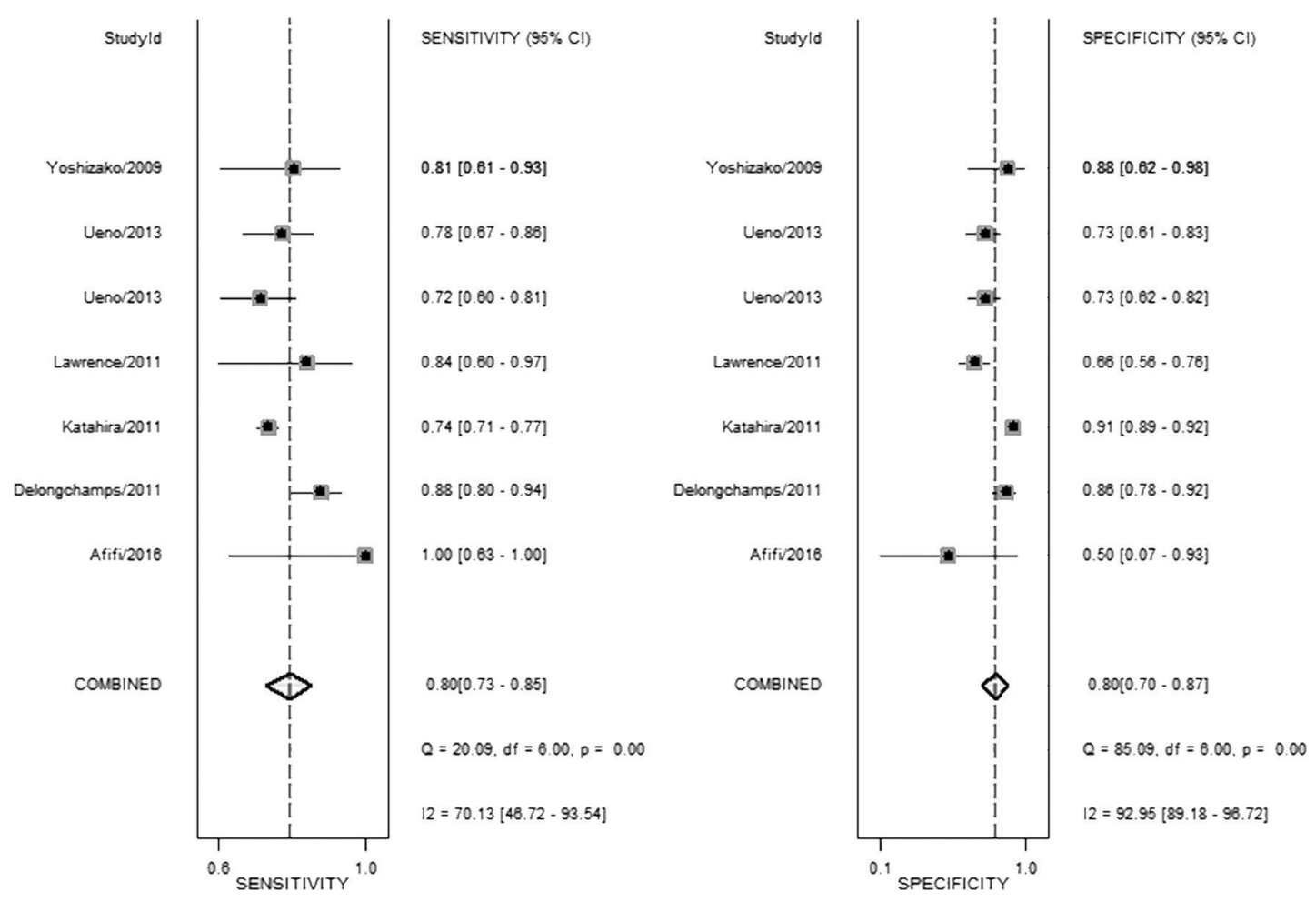

Figure 7 Coupled forest plots show pooled estimates of sensitivity and specificity of biparametric MRI for cancer located at transition zone. 

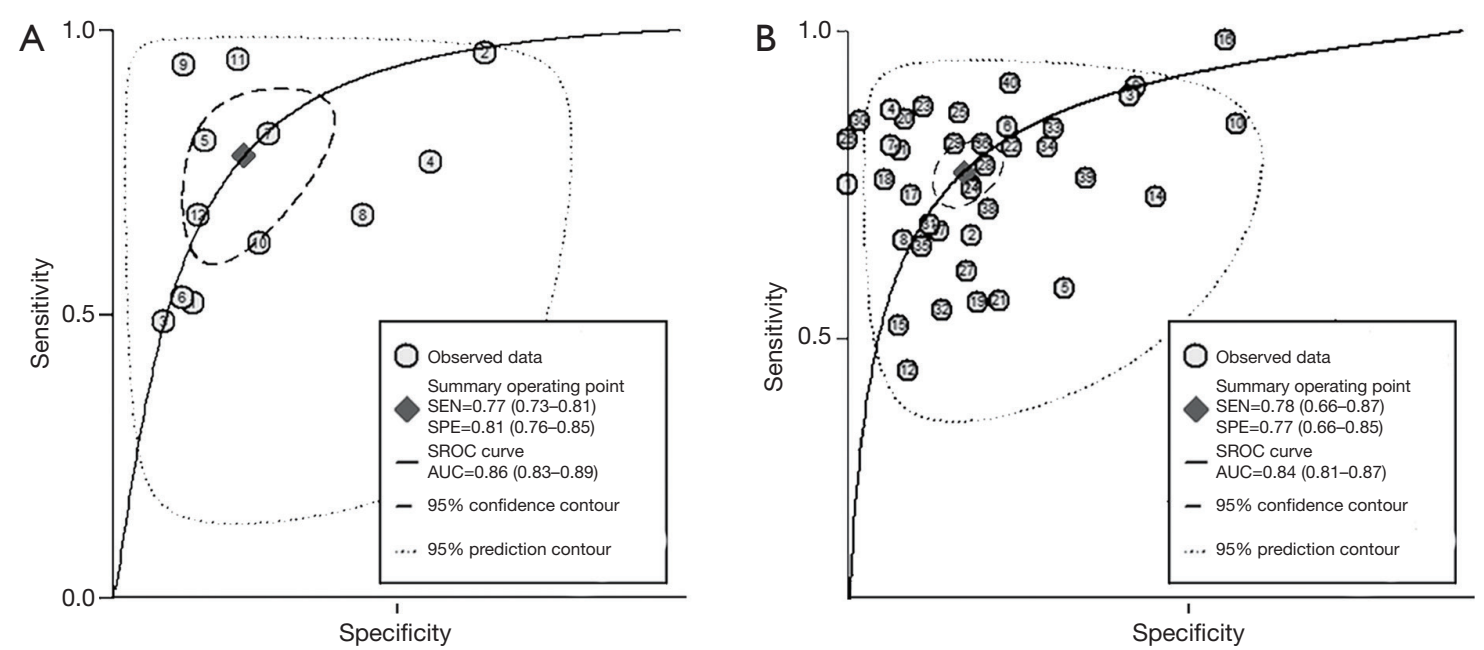

Figure 8 Summary ROC (SROC) curves with prediction and confidence contours of biparametric MRI for overall cancer (A) and clinically significant cancer (B).
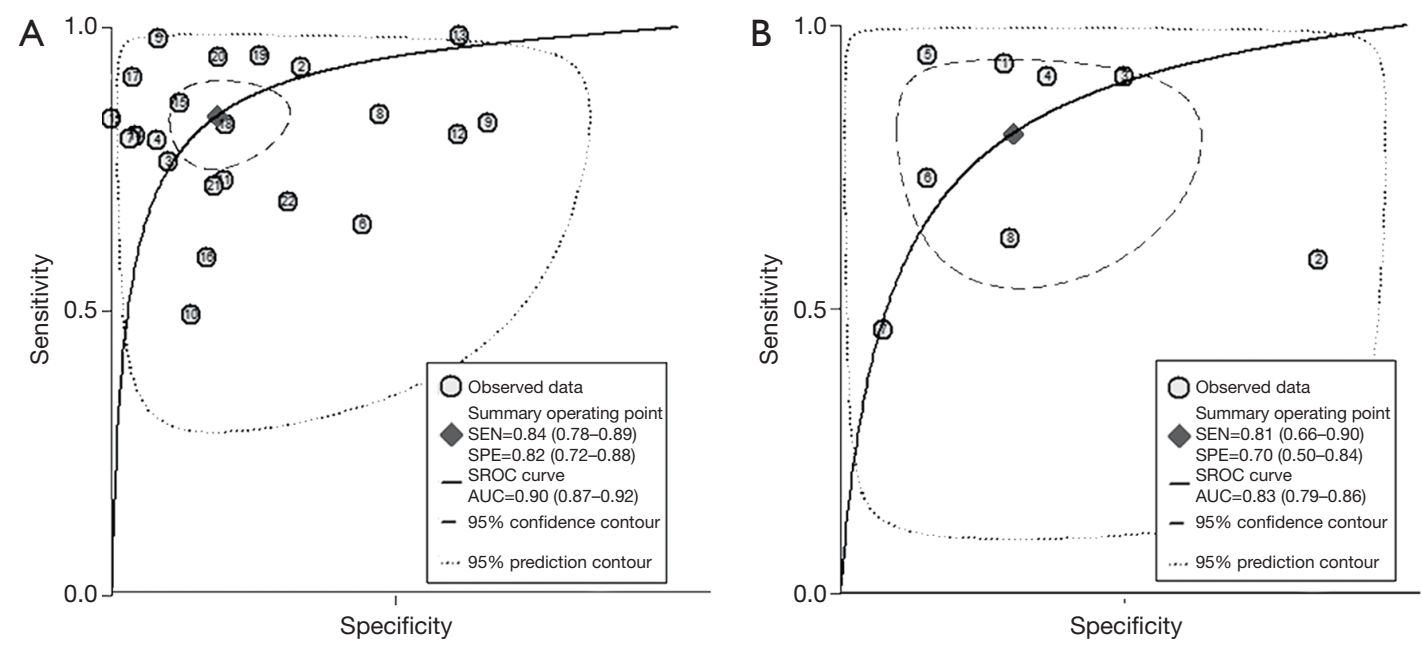

Figure 9 Summary ROC (SROC) curves with prediction and confidence contours of multiparametric MRI for overall cancer (A) and for clinically significant cancer (B).

result would be a true negative, thus reducing the likelihood of additional intervention such as prostate biopsy, the 7\% lower sensitivity of bpMRI may be an acceptable tradeoff for lower potential risk of adverse effects and therapy cost. Besides, the relatively low sensitivity of bpMRI could be fixed through combining with other clinical indicators. Boesen et al. (60) revealed positive potential for a model combining bpMRI and prostate-specific antigen density (PSAD) for detection of PCa among 808 biopsy-naïve men. Knaapila et al. (61) indicated PSAD could improve the NPV among men with equivocal suspicion on bpMRI, this imaging criteria coupled as an adjunct with PSA level and PSAD, could provide even more accuracy in detecting csPCa. Moreover, the issue of access to MRI caused by limited availability may be remedied through the shorter acquisition time (62). Given the impressive specificity and sensitivity of bpMRI, it may be considered as a pre-biopsy test for $\mathrm{PCa}$, in place of mpMRI.

Three systematic reviews (including two meta-analyses regarding) which explored the role of mpMRI in localized PCa have been published recently. In the study by Niu et al. (63) which evaluated 33 studies using a combination of T2WI, DWI, the pooled sensitivity and specificity were 0.81 (95\% CI: $0.76-0.85$ ) and 0.77 (95\% CI: 0.69-0.84), 

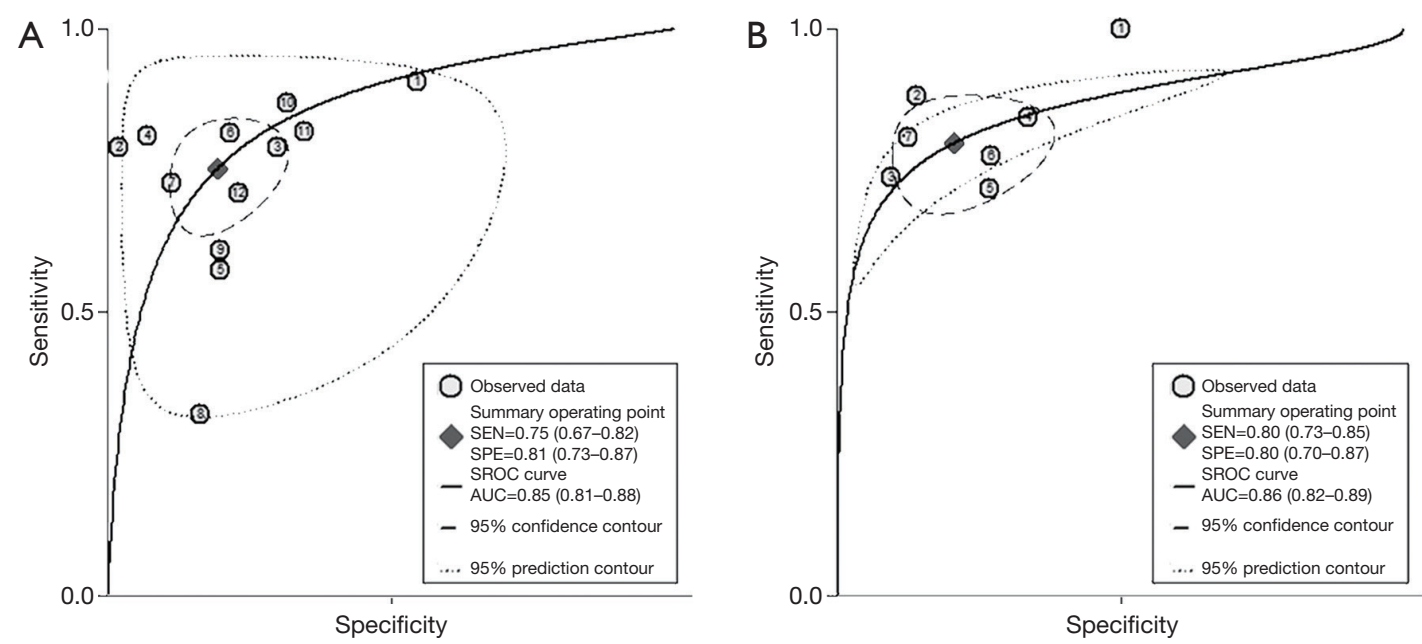

Figure 10 Summary ROC (SROC) curves with prediction and confidence contours of biparametric MRI for cancer located at peripheral zone (A) and transition zone (B).

Table 3 Subgroup analysis of analysis

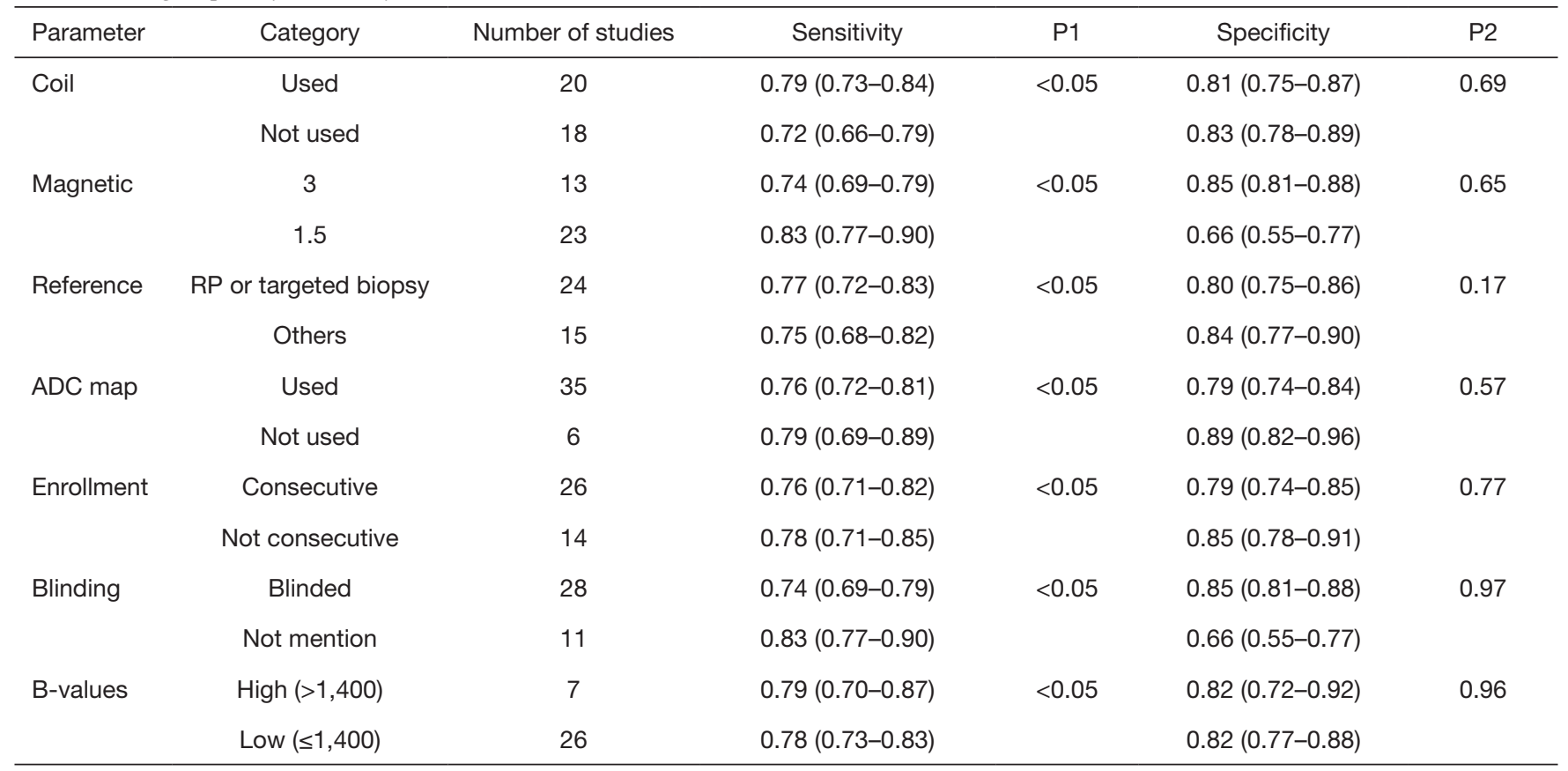

respectively. In a more recent meta-analysis by Woo et al. (6) which analyzed 20 studies, the pooled sensitivity and specificity were 0.74 (95\% CI: 0.66-0.81) and 0.90 (95\% CI: $0.87-0.93)$, respectively. Compared with the former review, the current study is the first meta-analysis to evaluate the performance of bpMRI based on different location of PCa, and assess their discrimination between bpMRI and mpMRI in the detection of csPCa.
From our present study, bpMRI may be sufficient and may not miss csPCa. The pooled specificity demonstrated no significant difference between bpMRI and mpMRI [bpMRI, 0.77 (95\% CI, 0.66-0.85); mpMRI, 0.70 (95\% CI, $0.50-0.84) ; \mathrm{P}=0.518]$. The pooled sensitivity also indicated little significant difference between these two groups [bpMRI, 0.78 (95\% CI, 0.66-0.87); mpMRI, 0.81 (95\% CI, $0.66-0.90) ; \mathrm{P}=0.135]$ (Figures 5,12). It means those tumors 

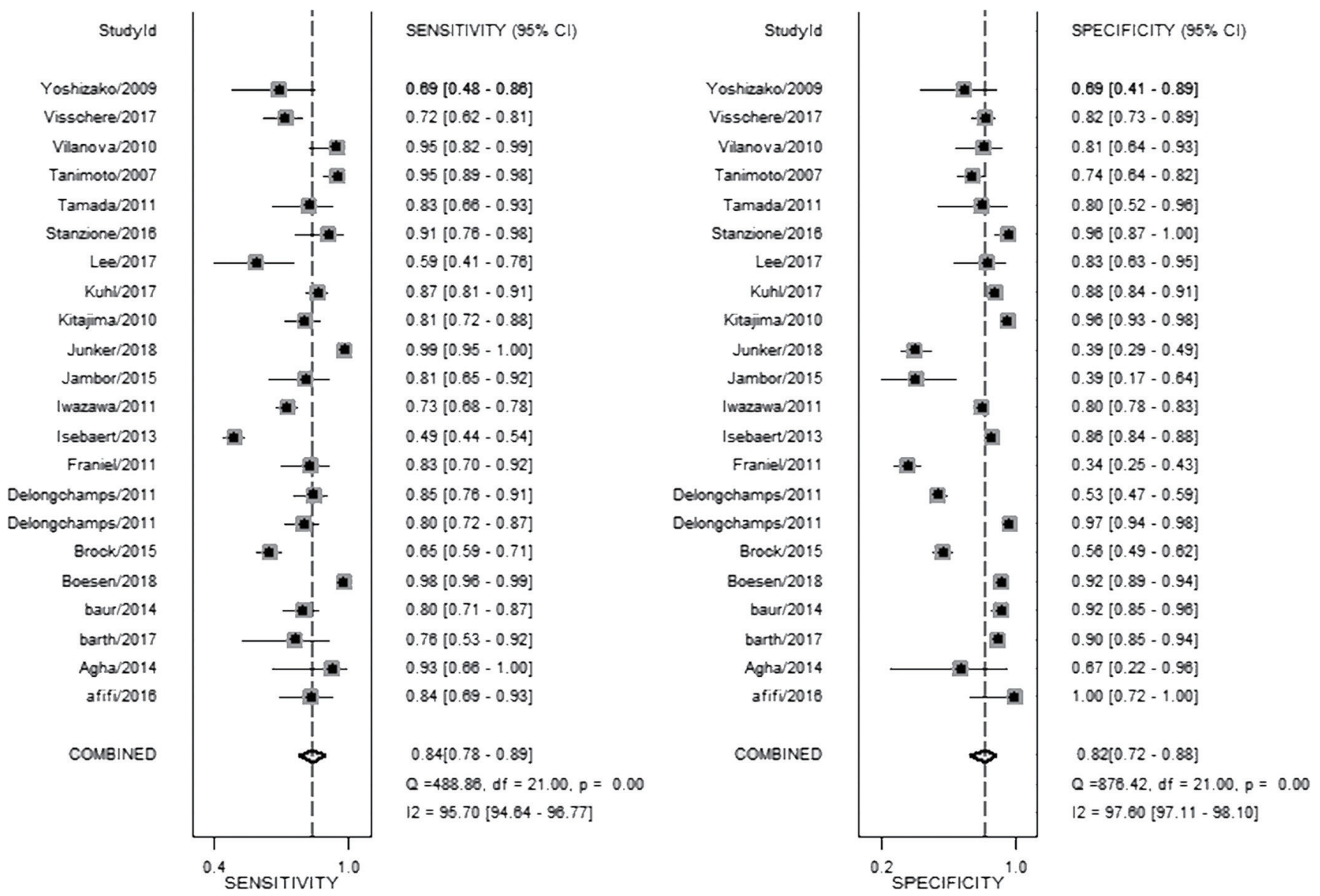

Figure 11 Coupled forest plots show pooled estimates of sensitivity and specificity of multiparametric MRI for overall cancer

ignored by bpMRI are mostly clinical insignificant and may also be ignored by mpMRI. Moreover, these tumors are more likely to remain latent in long-term follow-up and active surveillance.

Barth et al. (20) suggested that for the diagnose of csPCa, there is no significant difference between the diagnostic performance of a bpMRI and mpMRI protocol, which met our results. Boesen et al. (8) demonstrated the high NPV of $\mathrm{bpMRI}$ in ruling out csPCa in biopsy-naive men, a simple, rapid bpMRI method could be used as a triage test to improve risk stratification and to exclude aggressive disease and avoid unnecessary biopsies. On the other hand, Greer et al. (9) indicated that adding DCE-MRI to DWI scores in the peripheral zone yielded meaningful progress for detecting csPCa. Although the application of bpMRI prior to biopsy could decrease the risk of over-biopsy, reduce rates of over-detection, future work must be finished for bpMRI towards maintaining the same high diagnostic yield of mpMRI without compromising oncologic outcomes and cancer detection.

Based on our current results, for the detection of cancer located at transitional zone, both the sensitivity and specificity did not demonstrate a significant difference between these two groups [sen: bpMRI, 0.80 (95\% CI, 0.73-0.85); mpMRI, 0.75 (95\% CI, 0.45-0.91); $\mathrm{P}=0.0845$,spe: bpMRI, 0.80 (95\% CI, 0.70-0.87); mpMRI, 0.86 (95\% CI, 0.74-0.93); $\mathrm{P}=0.0982] \mathrm{DWI}$ alone is enough for cancer located in transitional zone which met the results of PI-RADSv2. While for the cancer located in peripheral zone, the pooled specificity demonstrated significant difference between bpMRI and mpMRI [bpMRI, 0.81 (95\% CI, 0.73-0.87); mpMRI, 0.96 (95\% CI, 0.92-0.98); $\mathrm{P}<0.05]$. The sensitivity, however, indicated little significant difference between these two groups [bpMRI, 0.75 (95\% CI, 0.67-0.82); mpMRI, 0.74 (95\% CI, 0.66-0.80); $\mathrm{P}=0.943]$. 

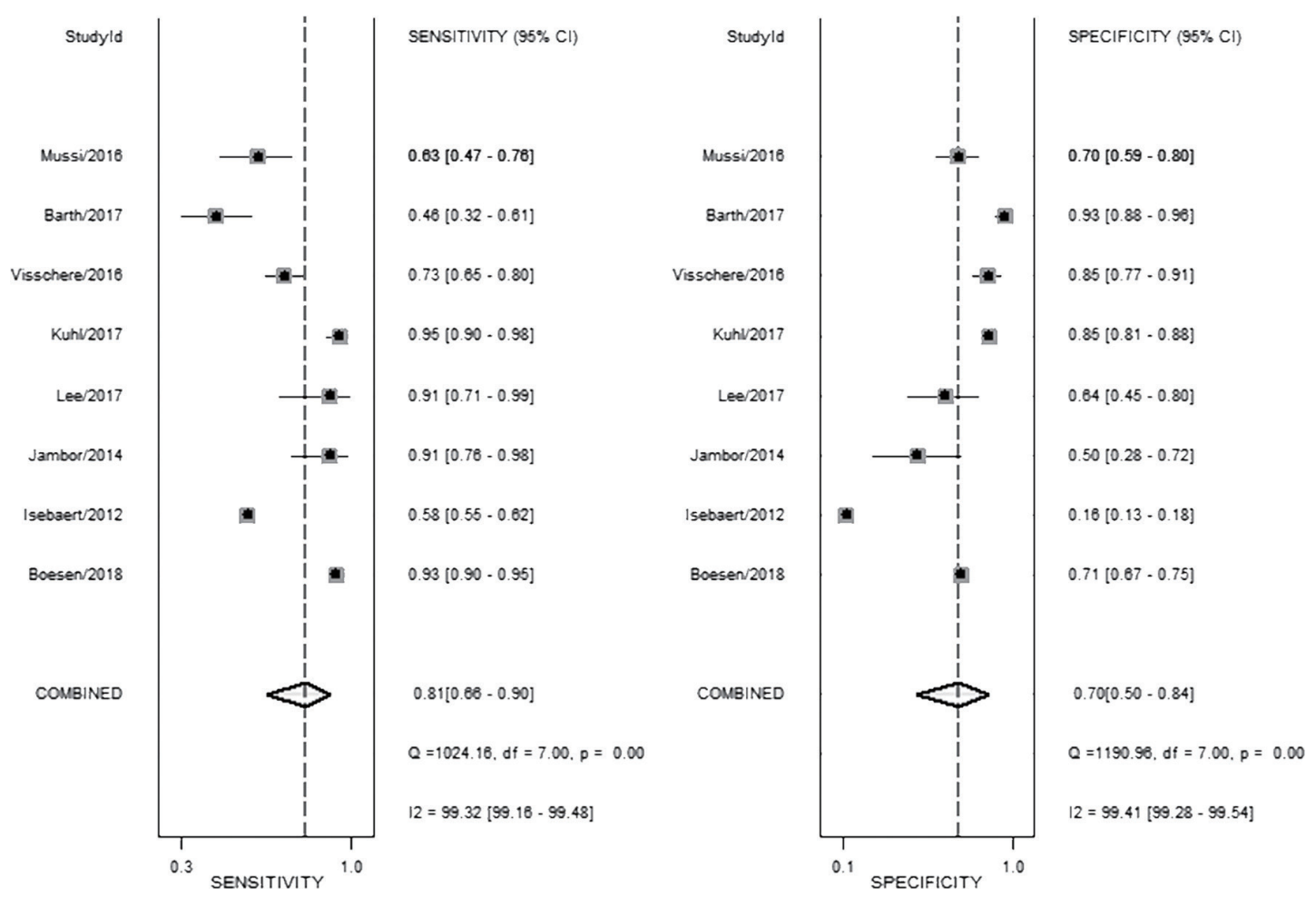

Figure 12 Coupled forest plots show pooled estimates of sensitivity and specificity of multiparametric MRI for clinically significant cancer.

From our analysis, the application of DCE contributes to unignorable improvements in specificity for peripheral PCa. Multiple studies have demonstrated that DCE-MRI can successfully detect $\mathrm{PCa}$ with a high sensitivity and specificity and help in tumor staging in peripheral zone (64-66). However, Delongchamps et al. (23) suggested DCEMRI may decrease the accuracy of T2WI and DWI for the cancer located at the central gland without significant improvement in peripheral zone. These debatable reports might be explained by different references to evaluate DCE-MRI in a quantitative way. After the PI-RADS score was updated in 2016 by ESUR and American College of Radiology (3), the question whether DCE-MRI could lead to an added value and better performance in the interpretation of mpMRI might be answered in the future.

The b-value is one of the significant factors that lead to the heterogeneity based on our subgroup analysis, it reflects the timings and strength of magnetic field gradients of DWI applied to the patient, and the collection of multiple b-values permits the calculation of ADC map. Currently, based on the PI-RADSV2, the recommended b-values is at least $1,400 \mathrm{~s} / \mathrm{mm}^{2}$, or if possible, up to $2,000 \mathrm{~s} / \mathrm{mm}^{2}$ (3). Our subgroup analysis demonstrated that high $\mathrm{b}$ values $\geq 1,400 \mathrm{~s} / \mathrm{mm}^{2}$ lead to significantly higher sensitivity and specificity for detecting $\mathrm{PCa}$, Therefore, forest plots were also accomplished in present study to make a comparison between mpMRI and bpMRI with high $b$ values $\geq 1,400 \mathrm{~s} / \mathrm{mm}^{2}$ (Figure 13). As shown in our results, there is no significant difference in both sensitivity [bpMRI with high b values 0.83 (95\% CI, 0.72-0.90); mpMRI 0.84 (95\% CI, 0.78-0.89), $\mathrm{P}=0.431]$ and specificity [bpMRI with high b values $0.78(95 \%$ CI, 0.63-0.88); mpMRI 0.82 (95\% CI, 0.72-0.88) $\mathrm{P}=0.621$ ] (Figures 11,13). The AUC is 0.88 which is similar to that of mpMRI (AUC =0.90) (Figures 9,14). Maas et al. (67) indicated that the application of high-b-value computed could avoid artefacts and improve lesion-to-background contrast ratios for the detection of PCa. Syer et al. (68) suggested that diagnostic accuracy of combined DWI and T2WI is trustable with high b-values improving sensitivity while maintaining specificity. Further large-scale studies specifically exploring the comparison between high b-value bpMRI and mpMRI should be made to acquire an exact result.

There are several potential limitations in our review. First, the included studies were heterogeneous in their methods, which affected the general applicability of the 

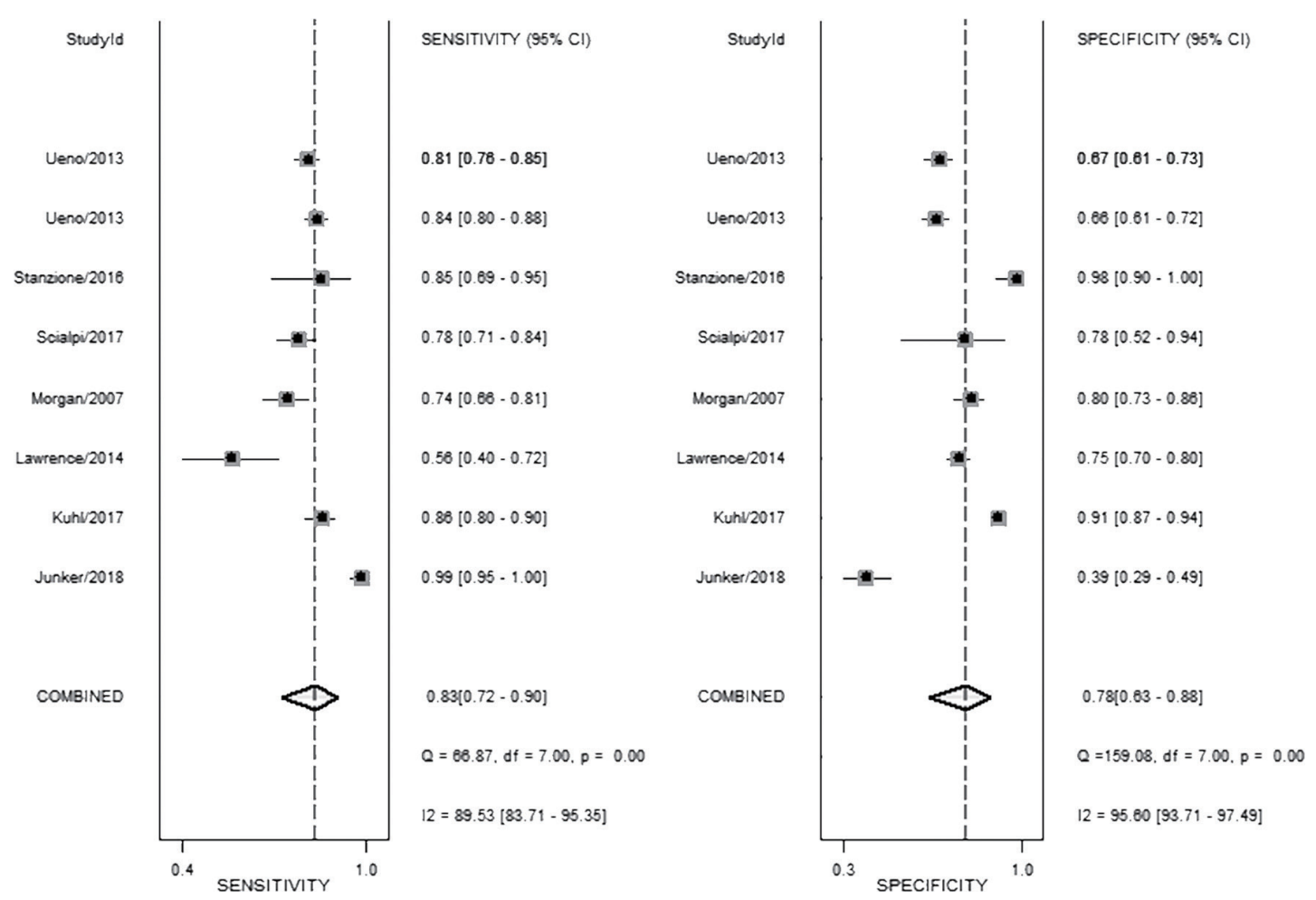

Figure 13 Coupled forest plots show pooled estimates of sensitivity and specificity of biparametric MRI combined with high b value MRI.

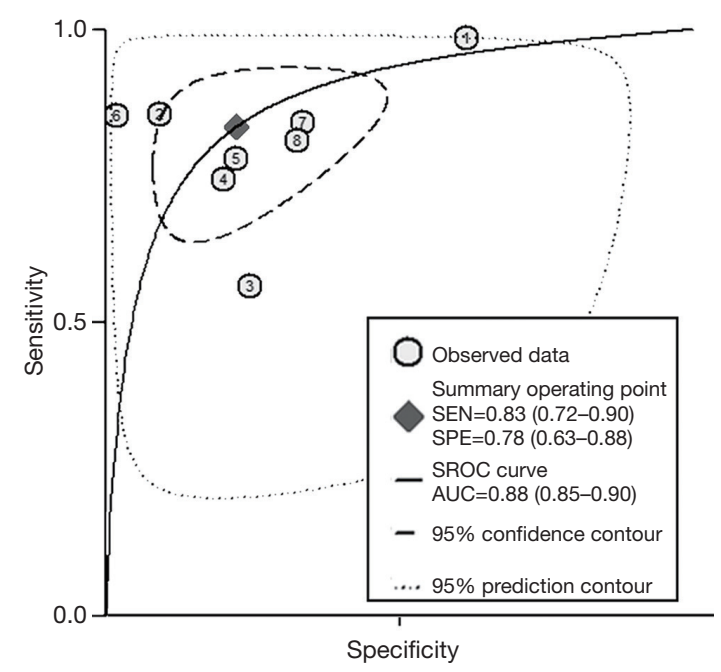

Figure 14 Summary ROC (SROC) curves with prediction and confidence contours of biparametric MRI combined with high $\mathrm{b}$ value MRI

summary estimates. To explore the heterogeneity of our data, we performed meta-regression and multiple subgroup analysis so that the diagnostic accuracy of bpMRI could be improved in the future. Second, until recently the definition of clinically relevant $\mathrm{PCa}$ varied considerably between each studies, which might have resulted in unreliable conclusions in our study. Third, studies with negative results are less likely to be published, which may lead to exaggeration of the beneficial effects in meta-analysis. Fourth, the different versions of PI-RADS score the included studies used may have an impact on our results. Finally, our meta-analysis focused on newly diagnosed or clinically suspected PCa. The results of our meta-analysis do not apply to detection or staging of recurrent $\mathrm{PCa}$.

\section{Conclusions}

A head-to-head comparison showed that the performance of bpMRI was similar to that of mpMRI for the diagnosis of PCa though the sensitivity was significantly lower. With the combination of high $\mathrm{b}$ value MRI, the sensitivity and specificity could improve to 0.83 and 0.78 respectively. The result of multiple subgroup analysis showed consistency with overall pooled estimates. 


\section{Acknowledgments}

Funding: None.

\section{Footnote}

Conflicts of Interest: All authors have completed the ICMJE uniform disclosure form (available at http://dx.doi. org/10.21037/tau.2020.02.03). The authors have no conflicts of interest to declare.

Ethical Statement: The authors are accountable for all aspects of the work in ensuring that questions related to the accuracy or integrity of any part of the work are appropriately investigated and resolved.

Open Access Statement: This is an Open Access article distributed in accordance with the Creative Commons Attribution-NonCommercial-NoDerivs 4.0 International License (CC BY-NC-ND 4.0), which permits the noncommercial replication and distribution of the article with the strict proviso that no changes or edits are made and the original work is properly cited (including links to both the formal publication through the relevant DOI and the license). See: https://creativecommons.org/licenses/by-nc$\mathrm{nd} / 4.0 \%$.

\section{References}

1. Todua F, Gagua R, Maglakelidze M, et al. Cancer incidence and mortality - Major patterns in GLOBOCAN 2012, worldwide and Georgia, 2015. Available online: http://science.org.ge/old/moambe/9-1/Todua\%20Fridon.

2. Haas GP, Delongchamps N, Brawley OW, et al. The worldwide epidemiology of prostate cancer: perspectives from autopsy studies. Can J Urol 2008;15:3866-71.

3. Weinreb JC, Barentsz JO, Choyke PL, et al. PI-RADS Prostate Imaging - Reporting and Data System: 2015, Version 2. Eur Urol 2016;69:16-40.

4. Johnson LM, Turkbey B, Figg WD, et al. Multiparametric MRI in prostate cancer management. Nat Rev Clin Oncol 2014;11:346-53.

5. Barentsz JO, Richenberg J, Clements R, et al. ESUR prostate MR guidelines 2012. Eur Radiol 2012;22:746-57.

6. Woo S, Suh CH, Kim SY, et al. Head-to-Head Comparison Between Biparametric and Multiparametric MRI for the Diagnosis of Prostate Cancer: A Systematic Review and Meta-Analysis. AJR Am J Roentgenol
2018;211:W226-41.

7. Kuhl CK, Bruhn R, Kramer N, et al. Abbreviated Biparametric Prostate MR Imaging in Men with Elevated Prostate-specific Antigen. Radiology 2017;285:493-505.

8. Boesen L, Norgaard N, Logager V, et al. Assessment of the Diagnostic Accuracy of Biparametric Magnetic Resonance Imaging for Prostate Cancer in Biopsy-Naive Men: The Biparametric MRI for Detection of Prostate Cancer (BIDOC) Study. JAMA Netw Open 2018;1:e180219.

9. Greer MD, Shih JH, Lay N, et al. Validation of the Dominant Sequence Paradigm and Role of Dynamic Contrast-enhanced Imaging in PI-RADS Version 2. Radiology 2017;285:859-69.

10. Puech P, Randazzo M, Ouzzane A, et al. How are we going to train a generation of radiologists (and urologists) to read prostate MRI? Curr Opin Urol 2015;25:522-35.

11. Vargas HA, Akin O, Franiel T, et al. Diffusion-weighted endorectal MR imaging at $3 \mathrm{~T}$ for prostate cancer: tumor detection and assessment of aggressiveness. Radiology 2011;259:775-84.

12. Aydin H, Hekimogl B, Tatar IG. Limitations, disabilities, and pitfalls of dynamic contrast-enhanced MRI as a diagnostic modality in prostate cancer. AJR Am J Roentgenol 2013;200:W326.

13. Berman RM, Brown AM, Chang SD, et al. DCE MRI of prostate cancer. Abdom Radiol (NY) 2016;41:844-53.

14. Ganeles J, Zollner A, Jackowski J, et al. Immediate and early loading of Straumann implants with a chemically modified surface (SLActive) in the posterior mandible and maxilla: 1-year results from a prospective multicenter study. Clin Oral Implants Res 2008;19:1119-28.

15. Wallace B, J Dahabreh I, Trikalinos T, et al. Closing the Gap between Methodologists and End-Users: R as a Computational Back-End. J Stat Softw 2011;49:1-15.

16. Reitsma JB, Glas AS, Rutjes AW, et al. Bivariate analysis of sensitivity and specificity produces informative summary measures in diagnostic reviews. J Clin Epidemiol 2005;58:982-90.

17. Higgins JP, Thompson SG, Deeks JJ, et al. Measuring inconsistency in meta-analyses. BMJ 2003;327:557-60.

18. Afifi A, Ali Ramadan A, Alabbady A, et al. Multiparametric MRI and PI-RADS (V1) scoring system: New inception in cancer prostate diagnosis to evaluate diagnostic performance of different score combinations. Egypt J Radiol Nucl Med 2016;47:1083-94.

19. Agha M, Eid A. 3 Tesla MRI surface coil: Is it sensitive for prostatic imaging. 2014. Available online: https://pdf. sciencedirectassets.com 
20. Barth BK, De Visschere PJL, Cornelius A, et al. Detection of Clinically Significant Prostate Cancer: Short Dual-Pulse Sequence versus Standard Multiparametric MR Imaging-A Multireader Study. Radiology 2017;284:725-36.

21. Brock M, Roghmann F, Sonntag C, et al. Fusion of Magnetic Resonance Imaging and Real-Time Elastography to Visualize Prostate Cancer: A Prospective Analysis using Whole Mount Sections after Radical Prostatectomy. Ultraschall Med 2015;36:355-61.

22. Delongchamps NB, Beuvon F, Eiss D, et al. Multiparametric MRI is helpful to predict tumor focality, stage, and size in patients diagnosed with unilateral low-risk prostate cancer. Prostate Cancer Prostatic Dis 2011;14:232-7.

23. Delongchamps NB, Rouanne M, Flam T, et al. Multiparametric magnetic resonance imaging for the detection and localization of prostate cancer: combination of T2-weighted, dynamic contrast-enhanced and diffusionweighted imaging. BJU Int 2011;107:1411-8.

24. Franiel T, Stephan C, Erbersdobler A, et al. Areas suspicious for prostate cancer: MR-guided biopsy in patients with at least one transrectal US-guided biopsy with a negative finding--multiparametric MR imaging for detection and biopsy planning. Radiology 2011;259:162-72.

25. Haider MA, van der Kwast TH, Tanguay J, et al. Combined T2-Weighted and Diffusion-Weighted MRI for Localization of Prostate Cancer. AJR Am J Roentgenol 2007;189:323-8.

26. Isebaert S, Van den Bergh L, Haustermans K, et al. Multiparametric MRI for prostate cancer localization in correlation to whole-mount histopathology. J Magn Reson Imaging 2013;37:1392-401.

27. Jambor I, Kahkonen E, Taimen P, et al. Prebiopsy multiparametric $3 \mathrm{~T}$ prostate MRI in patients with elevated PSA, normal digital rectal examination, and no previous biopsy. J Magn Reson Imaging 2015;41:1394-404.

28. Kitamura K, Muto S, Yokota I, et al. Feasibility of multiparametric prostate magnetic resonance imaging in the detection of cancer distribution: histopathological correlation with prostatectomy specimens. Prostate Int 2014;2:188-95.

29. Lee DH, Nam JK, Lee SS, et al. Comparison of Multiparametric and Biparametric MRI in First Round Cognitive Targeted Prostate Biopsy in Patients with PSA Levels under 10 ng/mL. Yonsei Med J 2017;58:994-9.

30. Morgan VA, Kyriazi S, Ashley SE, et al. Evaluation of the potential of diffusion-weighted imaging in prostate cancer detection. Acta Radiol 2007;48:695-703.
31. Rinaldi D, Fiocchi F, Ligabue G, et al. Role of diffusionweighted magnetic resonance imaging in prostate cancer evaluation. Radiol Med 2012;117:1429-40.

32. Rais-Bahrami S, Siddiqui MM, Vourganti S, et al. Diagnostic value of biparametric magnetic resonance imaging (MRI) as an adjunct to prostate-specific antigen (PSA)-based detection of prostate cancer in men without prior biopsies. BJU Int 2015;115:381-8.

33. Baur AD, Maxeiner A, Franiel T, et al. Evaluation of the prostate imaging reporting and data system for the detection of prostate cancer by the results of targeted biopsy of the prostate. Invest Radiol 2014;49:411-20.

34. Doo KW, Sung DJ, Park BJ, et al. Detectability of low and intermediate or high risk prostate cancer with combined T2-weighted and diffusion-weighted MRI. Eur Radiol 2012;22:1812-9.

35. Fascelli M, Rais-Bahrami S, Sankineni S, et al. Combined Biparametric Prostate Magnetic Resonance Imaging and Prostate-specific Antigen in the Detection of Prostate Cancer: A Validation Study in a Biopsy-naive Patient Population. Urology 2016;88:125-34.

36. Iwazawa J, Mitani T, Sassa S, et al. Prostate cancer detection with MRI: is dynamic contrast-enhanced imaging necessary in addition to diffusion-weighted imaging? Diagn Interv Radiol 2011;17:243-8.

37. Jung SI, Donati OF, Vargas HA, et al. Transition Zone Prostate Cancer: Incremental Value of Diffusion-weighted Endorectal MR Imaging in Tumor Detection and Assessment of Aggressiveness. Radiology 2013;269:493-503.

38. Junker D, Steinkohl F, Fritz V, et al. Comparison of multiparametric and biparametric MRI of the prostate: are gadolinium-based contrast agents needed for routine examinations? World J Urol 2019;37:691-9.

39. Katahira K, Takahara T, Kwee TC, et al. Ultrahigh-b-value diffusion-weighted MR imaging for the detection of prostate cancer: evaluation in 201 cases with histopathological correlation. Eur Radiol 2011;21:188-96.

40. Kitajima K, Kaji Y, Fukabori Y, et al. Prostate cancer detection with 3 T MRI: comparison of diffusionweighted imaging and dynamic contrast-enhanced MRI in combination with T2-weighted imaging. J Magn Reson Imaging 2010;31:625-31.

41. Lawrence EM, Tang SY, Barrett T, et al. Prostate cancer: performance characteristics of combined T(2)W and DWMRI scoring in the setting of template transperineal re-biopsy using MR-TRUS fusion. Eur Radiol 2014;24:1497-505.

42. Lim HK, Kim JK, Kim KA, et al. Prostate cancer: apparent diffusion coefficient map with T2-weighted images for 
detection--a multireader study. Radiology 2009;250:145-51.

43. Mussi TC, Martins T, Garcia RG, et al. Are Dynamic Contrast-Enhanced Images Necessary for Prostate Cancer Detection on Multiparametric Magnetic Resonance Imaging? Clin Genitourin Cancer 2017;15:e447-54.

44. Naiki T, Okamura T, Nagata D, et al. Preoperative prediction of neurovascular bundle involvement of localized prostate cancer by combined T2 and diffusionweighted imaging of magnetic resonance imaging, number of positive biopsy cores, and Gleason score. Asian Pac J Cancer Prev 2011;12:909-13.

45. Rosenkrantz AB, Mannelli L, Kong X, et al. Prostate cancer: utility of fusion of T2-weighted and high b-value diffusion-weighted images for peripheral zone tumor detection and localization. J Magn Reson Imaging 2011;34:95-100.

46. Scialpi M, Prosperi E, D'Andrea A, et al. Biparametric versus Multiparametric MRI with Non-endorectal Coil at $3 \mathrm{~T}$ in the Detection and Localization of Prostate Cancer. Anticancer Res 2017;37:1263-71.

47. Schimmöller L, Quentin M, Arsov C, et al. MR-sequences for prostate cancer diagnostics: validation based on the PIRADS scoring system and targeted MR-guided in-bore biopsy. Eur Radiol 2014;24:2582-9.

48. Shimofusa R, Fujimoto H, Akamata H, et al. Diffusionweighted imaging of prostate cancer. J Comput Assist Tomogr 2005;29:149-53.

49. Stanzione A, Imbriaco M, Cocozza S, et al. Biparametric $3 \mathrm{~T}$ Magnetic Resonance Imaging for prostatic cancer detection in a biopsy-naive patient population: a further improvement of PI-RADS v2? Eur J Radiol 2016;85:2269-74.

50. Tamada T, Sone T, Higashi H, et al. Prostate cancer detection in patients with total serum prostate-specific antigen levels of 4-10 ng/mL: diagnostic efficacy of diffusion-weighted imaging, dynamic contrast-enhanced MRI, and T2-weighted imaging. AJR Am J Roentgenol 2011;197:664-70.

51. Tanimoto A, Nakashima J, Kohno H, et al. Prostate cancer screening: The clinical value of diffusion-weighted imaging and dynamic MR imaging in combination with T2weighted imaging. J Magn Reson Imaging 2007;25:146-52.

52. Thestrup KC, Logager V, Baslev I, et al. Biparametric versus multiparametric MRI in the diagnosis of prostate cancer. Acta Radiol Open 2016;5:2058460116663046.

53. Ueno Y, Kitajima K, Sugimura K, et al. Ultra-high b-value diffusion-weighted MRI for the detection of prostate cancer with 3-T MRI. J Magn Reson Imaging 2013;38:154-60.
54. Ueno Y, Takahashi S, Kitajima K, et al. Computed diffusion-weighted imaging using 3-T magnetic resonance imaging for prostate cancer diagnosis. Eur Radiol 2013;23:3509-16.

55. Vilanova JC, Barcelo-Vidal C, Comet J, et al. Usefulness of prebiopsy multifunctional and morphologic MRI combined with free-to-total prostate-specific antigen ratio in the detection of prostate cancer. AJR Am J Roentgenol 2011;196:W715-22.

56. De Visschere P, Lumen N, Ost P, et al. Dynamic contrastenhanced imaging has limited added value over T2weighted imaging and diffusion-weighted imaging when using PI-RADSv2 for diagnosis of clinically significant prostate cancer in patients with elevated PSA. Clin Radiol 2017;72:23-32.

57. Yağci AB, Ozari N, Aybek Z, et al. The value of diffusionweighted MRI for prostate cancer detection and localization. Diagn Interv Radiol 2011;17:130-4.

58. Yoshimitsu K, Kiyoshima K, Irie H, et al. Usefulness of apparent diffusion coefficient map in diagnosing prostate carcinoma: correlation with stepwise histopathology. J Magn Reson Imaging 2008;27:132-9.

59. Yoshizako T, Wada A, Hayashi T, et al. Usefulness of Diffusion-Weighted Imaging and Dynamic ContrastEnhanced Magnetic Resonance Imaging in the Diagnosis of Prostate Transition-Zone Cancer. Acta Radiol 2008;49:1207-13.

60. Boesen L, Norgaard N, Logager V, et al. Prebiopsy Biparametric Magnetic Resonance Imaging Combined with Prostate-specific Antigen Density in Detecting and Ruling out Gleason 7-10 Prostate Cancer in Biopsy-naive Men. Eur Urol Oncol 2019;2:311-9.

61. Knaapila J, Jambor I, Perez IM, et al. Prebiopsy IMPROD Biparametric Magnetic Resonance Imaging Combined with Prostate-Specific Antigen Density in the Diagnosis of Prostate Cancer: An External Validation Study. Eur Urol Oncol 2019. [Epub ahead of print].

62. Gayet M, van der Aa A, Beerlage HP, et al. The value of magnetic resonance imaging and ultrasonography (MRI/ US)-fusion biopsy platforms in prostate cancer detection: a systematic review. BJU Int 2016;117:392-400.

63. Niu XK, Chen XH, Chen ZF, et al. Diagnostic Performance of Biparametric MRI for Detection of Prostate Cancer: A Systematic Review and Meta-Analysis. AJR Am J Roentgenol 2018;211:369-78.

64. Hara N, Okuizumi M, Koike H, et al. Dynamic contrastenhanced magnetic resonance imaging (DCE-MRI) is a useful modality for the precise detection and staging of 
early prostate cancer. Prostate 2005;62:140-7.

65. Kozlowski P, Chang SD, Jones EC, et al. Combined diffusion-weighted and dynamic contrast-enhanced MRI for prostate cancer diagnosis--correlation with biopsy and histopathology. J Magn Reson Imaging 2006;24:108-13.

66. Franiel T, Ludemann L, Rudolph B, et al. Evaluation of normal prostate tissue, chronic prostatitis, and prostate cancer by quantitative perfusion analysis using a dynamic contrast-enhanced inversion-prepared dual-contrast

Cite this article as: Liang Z, Hu R, Yang Y, An N, Duo X, Liu Z, Shi S, Liu X. Is dynamic contrast enhancement still necessary in multiparametric magnetic resonance for diagnosis of prostate cancer: a systematic review and meta-analysis. Transl Androl Urol 2020;9(2):553-573. doi: 10.21037/tau.2020.02.03 gradient echo sequence. Invest Radiol 2008;43:481-7.

67. Maas MC, Futterer JJ, Scheenen TW. Quantitative evaluation of computed high B value diffusion-weighted magnetic resonance imaging of the prostate. Invest Radiol 2013;48:779-86.

68. Syer TJ, Godley KC, Cameron D, et al. The diagnostic accuracy of high b-value diffusion- and T2-weighted imaging for the detection of prostate cancer: a metaanalysis. Abdom Radiol (NY) 2018;43:1787-97. 